\title{
Isoprenoid-mediated changes in the glycero- phospholipid molecular species of the sterol auxotrophic fungus Lagenidium giganteum
}

\author{
James L. Kerwin, Amy R. Tuininga, Alison M. Wiens, Jennifer C. Wang, \\ Jessica J. Torvik, Misty L. Conrath and Joanna K. MacKichan
}

Author for correspondence: James L. Kerwin. Tel: +1 206543 9737. Fax : +1 2065433262.

Botany Department AJ-30, University of Washington, Seattle, WA 98195, USA
The mosquito pathogenic fungus Lagenidium giganteum (Oomycetes: Lagenidiales) is a sterol auxotroph that can grow vegetatively in the absence of these compounds, but requires an exogenous source of sterols to enter its sexual and asexual reproductive cycles. Electrospray mass spectrometry (MS) and electrospray MS/MS were used to examine three major glycerophospholipid molecular species - glycerophosphocholine (GPC), glycerophosphoethanolamine (GPE) and glycerophosphoinositol (GPI) - from fungal mycelium and nuclei grown in defined medium with and without isoprenoids which induce (cholesterol and ergosterol) or do not induce (squalene, cholestane) reproduction. Testosterone supplementation of defined media inhibited growth of $L$. giganteum, so the effect of this steroid on phospholipid metabolism could not be assessed. Mycelium grown in defined media supplemented with these isoprenoids produced significantly different quantities of total phospholipid relative to unsupplemented media and to each other, ranging from a mean of $292 \mu \mathrm{g}$ phosphate per $g$ wet weight for cholesterol-supplemented media to $56 \mu \mathrm{g}$ phosphate per $\mathrm{g}$ wet weight for mycelium grown in the presence of squalene. A very large percentage of the GPC (69-80 mol\%) and GPI (74-79 mol\%) molecular species from mycelia and nuclei contained ether linkages. GPE molecular species had 13-20 mol \% ethercontaining moieties. The elevated levels of ether lipids may be related to the sterol auxotrophic nature of the fungus. Isoprenoid supplementation of defined growth media resulted in many significant changes in molecular species for all three lipid classes. Significant differences $(P<0.05)$ in the percentage of total cell ether lipids in GPC and GPE were generated by isoprenoid supplements to culture media. Mycelium grown in the presence of the two sterols which induce asexual and sexual reproduction in L. giganteum, cholesterol and ergosterol, had a significantly greater percentage of ethercontaining GPE moieties. The glycerolipid species from nuclei isolated from cultures grown with cholesterol and ergosterol were similar to the composition of nuclei isolated from fungus cultured in defined medium without any supplement or supplemented with squalene. The nuclear membrane from mycelia grown in cholestane-supplemented media, however, had a very different glycerophospholipid composition relative to either whole cells or nuclei from cells grown on other media. It appears that one of the reasons that cyclic isoprenoids such as cholestane do not induce fungal reproduction is that they drastically alter the nuclear membrane glycerophospholipid composition.

Keywords : Lagenidium giganteum, glycerophospholipids, sterol auxotroph, isoprenoids, nuclei 


\section{INTRODUCTION}

Sterols are a basic component of eukaryotic cells and have structural and regulatory roles in cell metabolism. They can make up $30 \mathrm{~mol} \%$ or more of the lipid fraction of some outer membranes (Yeagle, 1989, 1990), a concentration at which they undoubtedly play an important structural role. In some instances this bulk structural role can be met by sterols with a range of structures (Rodriguez et al., 1985). In contrast, the regulatory functions of sterols require very specific ring and side-chain structures and stereochemistry (Pinto et al., 1983; Bloch, 1981). In mammals the regulatory functions are satisfied by cholesterol (Bloch, 1983; Yeagle, 1989), while in yeast and many other fungi ergosterol is required (Rodriguez et al., 1982; Rodriguez \& Parks, 1983; Van den Bossche, 1990). These latter requirements can often be satisfied by sterol concentrations several orders of magnitude less than those required for their structural roles.

The roles of sterols in growth and morphogenesis have also been investigated in sterol auxotrophic members of the Oomycetes. These fungi can grow vegetatively in the absence of sterols, but require an exogenous source of these compounds to enter their sexual reproduction cycle (Elliot, 1983). Although they have long been regarded as a primitive group of fungi, Oomycetes have recently been more closely allied with heterokont algae in the Kingdom Chromista (Cavalier-Smith, 1989; Barr, 1992). This may explain in part why sterol structural requirements for the regulatory function in sterol auxotrophic Oomycetes are different from those of most other fungi. The developmental event with the strictest structural requirements is the production of oospores, the sexual stage of these organisms; however, structurally diverse sterols such as $\Delta-5$ (cholesterol, sitosterol), $\Delta-5,7$ (ergosterol), $\Delta-5,22$ (stigmasterol) and $\Delta-5,24$ (fucosterol, avenasterol) (Elliot, 1972, 1983) can, albeit with varying efficiency, induce oosporogenesis. The mechanism of sterol-induced reproduction in these fungi is not known, although sterols appear to play a pivotal role at the onset of meiosis (Elliot \& Sansome, 1977).

Lagenidium giganteum (Oomycetes: Lagenidiales), a facultative parasite of mosquito larvae, is a member of this primitive group of fungi which cannot synthesize sterols (Warner \& Domnas, 1981; Domnas \& Warner, 1991). It can grow vegetatively in the absence of sterols, but requires an exogenous source of these compounds to enter its asexual (Domnas et al., 1977) or sexual (Kerwin \& Washino, 1983) reproductive cycles. It is interesting that the mosquito hosts of L. giganteum also lack the ability to synthesize sterols, which are required for larval growth and development. Mosquito larvae obtain their sterols from the diet on which they are reared. These dietary sterols, following uptake and metabolism by the larvae, are then used by the fungus to promote its sexual and asexual reproduction. Both reproductive stages culminate in the production of motile zoospores, the infective stage of L. giganteum, and allow it to persist for weeks or years following its introduction into a suitable natural habitat.
Supplementation of growth media with specific phospholipids enhances oosporogenesis by L. giganteum (Kerwin $e t$ al., 1991) and other sterol auxotrophic Oomycetes (Ko, 1986; Kerwin \& Duddles, 1989). For some species there is a synergistic effect between phospholipids and sterols on oosporogenesis. Interactions between sterols and phospholipids have been examined in many artificial and biological membranes (Chia et al., 1993; Davidson et al., 1993). Sterol modulation of the fluidity of phospholipid acyl chains is well documented, but since microgram per $\mathrm{ml}$ quantities of these compounds in culture media also supplemented with phospholipids can exert profound developmental changes in sterol auxotrophic Oomycetes, at least some of their effects are not due to changes in bulk membrane properties.

The action of steroid hormones in regulating sexual reproduction by Acblya spp., which are Oomycetes capable of synthesizing sterols and related isoprenoids, has been extensively investigated (Barksdale, 1969; Horgen et al., 1983; Brunt \& Silver, 1991; McMorris et al., 1993). Although the possible role of sterols as steroid hormone precursors in sterol auxotrophic Oomycetes is the subject of controversy (Elliot, 1983), these compounds or their metabolites must directly or indirectly be involved in events in the nucleus associated with developmental regulation. The mechanism of sterol-induced reproduction in Oomycetes unable to synthesize sterols is not known, but this class of lipid also appears to play a pivotal role at the onset of meiosis (Elliot \& Sansome, 1977). One possible mechanism of isoprenoid-induced reproduction is to regulate the composition of glycerophospholipid molecular species in the nuclear membrane. Coordinated regulation of the nuclear composition of different lipid classes has been shown to occur in mammalian nuclei following dietary supplementation with highly polyunsaturated fatty acids (Chapkin et al., 1992). Sterols and other isoprenoids can alter membrane composition independently of developmentally regulated changes. As the initial step in understanding the role of isoprenoids in fungal morphogenesis, we have investigated the effects of isoprenoid supplementation of culture media on whole cell and nuclear glycerophospholipids from vegetative cultures of L. giganteum.

\section{METHODS}

Source and maintenance of fungal cultures. The California isolate of L. giganteum (ATCC 52675) was maintained on the following completely defined medium, designated DM2 (g per litre distilled water): glucose $4 \cdot 0$, sodium glutamate $2 \cdot 0$, asparagine $1 \cdot 0, \mathrm{Na}_{2}$ EDTA $0 \cdot 05, \mathrm{KH}_{2} \mathrm{PO}_{4} 0 \cdot 05, \mathrm{~K}_{2} \mathrm{HPO}_{4} 0 \cdot 05$, thiamin. $\mathrm{HCl} 0 \cdot 1, \mathrm{KNO}_{3} 0 \cdot 1$, methionine $0 \cdot 05, \mathrm{MgSO}_{4} .7 \mathrm{H}_{2} \mathrm{O}$ $0 \cdot 1, \mathrm{CaCl}_{2} \cdot 2 \mathrm{H}_{2} \mathrm{O} 0 \cdot 15, \mathrm{Fe}\left(\mathrm{NO}_{3}\right)_{2} \cdot 9 \mathrm{H}_{2} \mathrm{O} 0 \cdot 0081, \mathrm{MnCl}_{2} \cdot 4 \mathrm{H}_{2} \mathrm{O}$ $0.036, \mathrm{ZnSO}_{4} .7 \mathrm{H}_{2} \mathrm{O} 0.01, \mathrm{H}_{3} \mathrm{BO}_{3} 0.063, \mathrm{CuSO}_{4} .5 \mathrm{H}_{2} \mathrm{O} 0.008$, $\left(\mathrm{NH}_{4}\right)_{6} \mathrm{Mo}_{7} \mathrm{O}_{24} \cdot 4 \mathrm{H}_{2} \mathrm{O} 0 \cdot 01, \mathrm{CoCl}_{2} \cdot 6 \mathrm{H}_{2} \mathrm{O} 0 \cdot 017,250 \mu$ l tergitol NP-40/ethanol $(1: 1, \mathrm{v} / \mathrm{v})$, adjusted to $\mathrm{pH} 7 \cdot 0$ with $1 \mathrm{M} \mathrm{NaOH}$. The tergitol/ethanol, which was used to enhance solubilization of added isoprenoids, was included in the control media to ensure that the observed changes in glycerophospholipid species were due to addition of the isopentenoids rather than to the 
detergent. DM2 is a variation of defined media previously used to grow Oomycetes (Gleason, 1968; Ko, 1986). The medium was supplemented with excess glucose, which inhibits both sexual and asexual reproduction (Domnas et al., 1977; Kerwin \& Washino, 1983), so that altered glycerophospholipid species were not induced by developmental changes. Isoprenoids were added to DM2 at a concentration of $50 \mathrm{mg} \mathrm{l}^{-1}$. All compounds were added prior to autoclaving except for ergosterol, which was added after sterilization to minimize breakdown of this sterol, which is relatively labile due to the presence of conjugated double bonds at the $\Delta-5$ and -7 positions. Cultures were grown in 11 of medium in 2.81 Fernbach flasks inoculated with $10 \mathrm{ml}$ of stock culture, at 120 r.p.m. on a rotary shaker at $23 \pm 3^{\circ} \mathrm{C}$. Cultures used for comparative lipid analyses were $6-8 \mathrm{~d}$ old, in the stationary phase of their development. Fungal cultures were maintained for at least three generations on a given medium before extraction of phospholipids for mass spectral analysis.

Whole cell phospholipid extraction and fractionation. Stationary phase cultures ( $6-8 \mathrm{~d}$ old $)$ were collected by filtration on $100 \mu \mathrm{m}$ nylon mesh and washed in $1 \%(\mathrm{v} / \mathrm{v})$ aqueous tergitol NP-40/ethanol $(1: 1, \mathrm{v} / \mathrm{v})$ followed by distilled water (Lorenz $e t$ al., 1986). Total cell lipid was extracted using slight modifications (Kerwin \& Duddles, 1989) of the method of Folch $e$ al. (1957). The extract was concentrated and the glycerophospholipids separated by thin layer chromatography (TLC) on silica gel $G$, using hexane/diethyl ether/acetic acid $(90: 10: 1$, by vol.) as the developing solvent. The glycerophospholipids were extracted from the origin using approximately $10 \mathrm{ml}$ chloroform/methanol $(2: 1, \mathrm{v} / \mathrm{v})$, filtered from the silica gel and stored in chloroform at $-20{ }^{\circ} \mathrm{C}$ until used for quantification or electrospray MS.

Phospholipid quantification. Total phospholipid was measured using the procedure of Mrsny et al. (1986). Following separation by TLC, the phospholipid fractions were dried then resuspended in $50 \mu \mathrm{l}$ water and $0.5 \mathrm{ml}$ perchloric acid. Samples were digested for a minimum of $6 \mathrm{~h}$ in open-top tubes at $130^{\circ} \mathrm{C}$. After digestion, $1 \mathrm{ml} 2.5 \%(\mathrm{w} / \mathrm{v}$ ) ammonium molybdate, $3 \mathrm{ml}$ distilled water and $0.5 \mathrm{ml} 10 \%(\mathrm{w} / \mathrm{v}$ ) ascorbic acid were added to the tubes, which were incubated at $37^{\circ} \mathrm{C}$ for $1 \mathrm{~h}$. Using $\mathrm{NaH}_{2} \mathrm{PO}_{4}$ to generate a standard curve, phosphomolybdate concentration was monitored at $820 \mathrm{~nm}$.

Purification of nuclei. Lysing solution (LS) was prepared using (per litre distilled water): $91.1 \mathrm{~g}$ sorbitol, $0.609 \mathrm{~g} \mathrm{MgCl}_{2}, 0.442 \mathrm{~g}$ $\mathrm{CaCl}_{2}, 0.372 \mathrm{~g}$ EDTA, $170 \mathrm{mg}$ PMSF, $150 \mathrm{mg}$ DTT and $5.0 \mathrm{ml}$ absolute ethanol in $100 \mathrm{ml}$ distilled water. DTT and PMSF were added just prior to use. Washing solution (WS) consisted of (per litre distilled water): $1.576 \mathrm{~g}$ Tris $/ \mathrm{HCl}$ and $1.015 \mathrm{~g}$ $\mathrm{MgCl}_{2} \cdot 6 \mathrm{H}_{2} \mathrm{O}$. Final washing solution (FWS) was prepared by adding $0.5 \mathrm{ml}$ of a solution of $0.023 \mathrm{~g} \mathrm{NaCl}(20 \mathrm{mM})$ and $10 \mathrm{mg}$ PMSF dissolved in $2.5 \mathrm{ml}$ ethanol to $20 \mathrm{ml}$ of WS.

LS, WS and FWS were placed at $-20^{\circ} \mathrm{C}$ until ice began to form, and then placed at $5^{\circ} \mathrm{C}$. Two $10 \mathrm{~cm}$ diameter Buchner funnels with six layers of miracloth were chilled at $5^{\circ} \mathrm{C}$. Mycelium from stationary phase cultures was filtered and washed in distilled water. Approximately $10 \mathrm{~g}$ of cells was placed in a chilled metal blender cup. In order to initially disperse the mycelium, $60-80 \mathrm{ml}$ of ice cold LS was added to the cup and blended for 15-30 s at the highest setting using a Virtis Tempest polytron. Liquid $\mathrm{N}_{2}(1-2 \mathrm{ml})$ was added to the mixture and homogenization continued until the mixture thawed, usually 1-2 min. The process was repeated with liquid $\mathrm{N}_{2}$ added as necessary for 6-8 min. When a minimum of $70 \%$ of the cells had been disrupted as determined microscopically, the lysate was filtered twice using the miracloth and the chilled Buchner funnels. The filtrate was poured into centrifuge tubes and centrifuged for $10 \mathrm{~min}$ at about $8000 \mathrm{~g}$. The supernatant was decanted and the pellet resuspended in about $8 \mathrm{ml}$ ice-cold WS. The pellet was centrifuged and washed twice more. A Percoll step gradient was prepared using $5 \mathrm{ml}$ of each of 50, 40,20 and $10 \%(\mathrm{v} / \mathrm{v})$ Percoll solutions diluted with LS. The pellet from the final wash was resuspended in approximately $1 \mathrm{ml}$ ice cold FWS, gently layered on top of the Percoll, and centrifuged at $4{ }^{\circ} \mathrm{C}$ for $15 \mathrm{~min}$ at $6000 \mathrm{~g}$. A small amount of each individual band from the gradient was carefully removed and placed on a microscope slide with a drop of acridine orange (prepared just prior to use by dissolving $10 \mathrm{mg}$ acridine orange in $10 \mathrm{ml}$ distilled water). Following examination of the individual bands under a Zeiss fluorescence microscope, the band containing purified nuclei was extracted with a Pasteur pipette and resuspended in a small volume of FWS in a $3 \mathrm{ml}$ Reacti-vial (Pierce Chemical Co.). The vial was centrifuged for $5-10 \mathrm{~min}$ at about $8000 \mathrm{~g}$, and the supernatant removed with a Pasteur pipette. The pellet was resuspended in $\mathrm{CHCl}_{3} / \mathrm{MeOH}(2: 1$, $\mathrm{v} / \mathrm{v}$ ) and stored at $-20{ }^{\circ} \mathrm{C}$ until mass spectral analysis. Some preparations were resuspended in $0 \cdot 1 \mathrm{M}$ Tricine buffer, $\mathrm{pH} 7 \cdot 8$, for marker enzyme assays.

In addition to microscopic confirmation of the purity of nuclear preparations, the following enzyme marker assays (Chapkin et al., 1992) were used to confirm the absence of contaminating organelles: 5 '-nucleotidase for plasma membranes (Michell \& Hawthorne, 1965), modified according to Chapkin et al. (1992), using the method of Fiske \& Subbarrow (1925) to quantify phosphate release; glucose-6-phosphatase for microsomes; and cytochrome $c$ oxidase for mitochondria (Smith, 1953). Pellets containing over 1000 -fold more nuclei than was used for each molecular species analysis were used for the enzymic assays to ensure that any contaminating organelles would be detected.

A representative nuclear preparation was examined by transmission electron microscopy. Nuclei were fixed in $3 \%(\mathrm{v} / \mathrm{v})$ glutaraldehyde in $1.0 \mathrm{M}$ phosphate buffer, $\mathrm{pH} 7.3$, for $4 \mathrm{~h}$, followed by $2 \%$ (w/v) $\mathrm{OsO}_{4}$ in $1.0 \mathrm{M}$ phosphate buffer, $\mathrm{pH} 7 \cdot 3$, for $1 \mathrm{~h}$. The organelles were embedded in Epon 812, stained for $1 \mathrm{~min}$ in uranyl acetate and then lead citrate, and viewed at $80 \mathrm{kV}$ using a JEOL 100B microscope.

Phospholipid extraction from nuclei. Several hours before mass spectral analysis, samples were removed from the freezer, and the nuclear pellet extracted by vortexing the $\mathrm{CHCl}_{3} / \mathrm{MeOH}$ mixture twice for $2 \mathrm{~min}$, with 2 min periods of cooling on ice between vortexing. Longer extraction times removed unidentified (non-glycerolipid) components which interfered with the mass spectral analyses. The vials were centrifuged for $5 \mathrm{~min}$ at about $8000 \mathrm{~g}$, the $\mathrm{CHCl}_{3} / \mathrm{MeOH}$ was removed under a stream of nitrogen, and the lipids resuspended in $5 \mathrm{mM}$ methanolic ammonium acetate for mass spectral analysis.

Electrospray mass spectrometry. The major glycerophospholipid molecular species were identified using a combination of positive and negative ion electrospray mass spectrometry (MS) and MS/MS as described by Kerwin et al. (1994). Chloroform was evaporated from stored samples and dissolved in $5 \mathrm{mM}$ methanolic ammonium acetate at approximately $0.5-1 \mathrm{ng} \mu \mathrm{l}^{-1}$. This concentration was such that the peaks corresponding to the glycerophospholipids from most samples could be collected in 5-10 scans with minimal folding over of the $[M+\mathrm{H}]^{+}$(or $M^{+}$or $[M+60]^{+}$for choline-containing species) peaks. Lipid samples were analysed on a triple-quadrupole Sciex API III instrument (PE/SCIEX). Samples were infused into the electrospray source via a $50 \mu \mathrm{m}$ i.d. fused silica transfer line using a Harvard Apparatus pump at $1-3 \mu \mathrm{min}^{-1}$. Positive ion 
MS and MS/MS was run with an orifice voltage of 70 or $80 \mathrm{~V}$. Negative ion electrospray MS and MS/MS used an orifice voltage from -70 to $-90 \mathrm{~V}$. The interface temperature was maintained at $52{ }^{\circ} \mathrm{C}$. For tandem mass spectrometry (MS/MS), precursor ions were selected with the first of three quadrupoles (Q1) for collision-induced dissociation with argon in the second quadrupole (Q2). The third quadrupole (Q3) was scanned with a mass step of $0.20 \mathrm{Da}$ and $1 \mathrm{~ms}$ per step. Parent ion transmission was maximized by reducing the resolution of Q1 to transmit a $2-3 \mathrm{~m} / \mathrm{z}$ window about the selected parent ion, and Q3 resolution was adjusted to approximately $50 \%$ valley between peaks $3 \mathrm{Da}$ apart. Spectra were collected and analysed using proprietary software from Sciex Corporation.

Percentage composition of molecular species was generated using the peak intensities of ions from positive ion electrospray MS, scanning from either $\mathrm{m} / \mathrm{z} 450$ or 600 to $\mathrm{m} / \mathrm{z} 1000$. In positive ion electrospray MS and MS/MS, ions generated by parent species occurred as $\left[M+\mathrm{H}^{+}\right]$adducts, except for cholinecontaining species. In the positive ion mode, diacylphosphatidylcholine species occurred as $\mathrm{M}^{+}$due to the quaternary ammonium present in the headgroup. Ether-containing glycerophosphocholine occurred primarily as $[M+60]^{+}$adducts due to association with acetate ion. Confirmation of $[M+60]^{+}$ was readily obtained using positive ion MS/MS. In the negative ion mode, all choline-containing species formed acetate adducts. When multiple molecular species were represented by a single peak in positive ion electrospray, negative ion MS/MS spectra were used to determine the approximate contribution of individual species to each peak by summing the respective contributions of constituent fatty acids. For example, the peak at $\mathrm{m} / \mathrm{z} 764$ in negative ion electrospray MS contained both $18: 1 / 20: 4$ and $18: 2 / 20: 3$ phosphatidylethanolamine (PE). The contribution by each species to the peak intensity was obtained by adding the signal intensities at $m / z 281$ and 303 for $18: 1 / 20: 4$ $\mathrm{PE}$ and at $\mathrm{m} / \mathrm{z} 279$ and 305 for $18: 2 / 20: 3 \mathrm{PE}$. For the few peaks that contained both diacyl and acyl-ether species, the peak intensity corresponding to the fatty acyl constituent in the $s n-2$ position of the ether lipids was doubled to obtain an approximate contribution since there was no signal generated by the ether-linked moiety (Kerwin et al., 1994).

Electrospray MS and MS/MS is a powerful and versatile technique for structural studies of glycerophospholipids, and it is probable that many or all of the unique species reported below for, e.g. the cholestane-supplemented nuclei, are produced by cells cultured in the other media. Since we collected hundreds of electrospray MS/MS spectra for this study, we had to limit the amount of time spent scanning each peak. The great majority of MS/MS spectra were collected in 15-30 s, and data for less than $10 \%$ of the peaks required $45-120$ s to obtain a reliable signal. We arbitrarily chose not to collect data for periods longer than $2 \mathrm{~min}$. Molecular species reported as $0 \%$ or not reported either were not present or made up an insignificant percentage (usually less than $0 \cdot 1 \mathrm{~mol} \%$ ) of a given class of glycerophospholipid.

Sterol extraction and gas chromatography (GC). Nuclei isolated from mycelial cultures grown with ergosterol, cholesterol or cholestane supplements were analysed for the presence of sterols. Nuclear pellets were suspended in $2 \mathrm{ml} 0.5 \%$ pyrogallol, $2 \mathrm{ml} 60 \%$ (w/v) $\mathrm{KOH}$, and $3 \mathrm{ml}$ methanol. The mixture was refluxed with boiling stones for $2 \mathrm{~h}$ at $70-80^{\circ} \mathrm{C}$, and the saponified lipids extracted three times with $10 \mathrm{ml}$ hexane. The pooled hexane extracts were dried and resuspended in a minimal volume of methanol for GC (Lorenz et al., 1986). GC of underivatized sterols was done with a Hewlett Packard 5890 series II gas chromatograph using a $15 \mathrm{~m}$ DB-5 (5\% phenyl, J\&W Scientific) column, $0.32 \mathrm{~mm}$ i.d. The injector temperature was $250^{\circ} \mathrm{C}$, the FID temperature $310^{\circ} \mathrm{C}$. An initial temperature of $200^{\circ} \mathrm{C}$ was held for $2 \mathrm{~min}$, then raised to $240^{\circ} \mathrm{C}$

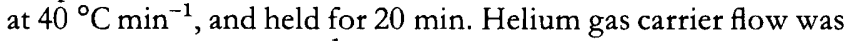
maintained at $2 \mathrm{ml} \mathrm{min}^{-1}$ by operating the electronic pressurecontrolled injector in the constant flow mode. Sterols were identified using relative retention times, with cholestane as the internal standard for ergosterol- and cholesterol-supplemented cultures, and cholesterol as the internal standard for cholestanesupplemented cultures. Sterol identification was confirmed using GC-MS.

The relative $\mathrm{mol} \%$ composition of glycerophospholipid molecular species is reported as the mean \pm 1 SD. Statistical differences were evaluated using Student's $t$-test. All statistical evaluations were done using the SYSTAT package.

\section{RESULTS}

\section{Total cell glycerophospholipids}

Isoprenoids alter the growth and morphogenesis of $L$. giganteum and related sterol auxotrophic Oomycetes. The compounds used in these investigations were chosen because of their effect on fungal development. Cholesterol and ergosterol can induce sexual and asexual reproduction, while cholestane and squalene, the acyclic precursor of sterols and related compounds, do not promote reproduction. The defined medium used in these investigations does not permit reproduction even with appropriate sterol supplementation, because of its high concentration of glucose. This medium was used so that observed changes in glycerophospholipid molecular species were not due to developmental changes.

One effect of isoprenoid supplementation was regulation of the amount of phospholipid produced by the fungus. In $\mu g$ phosphate per $g$ wet weight, mycelia cultured on the different media produced: defined medium (DM2), $164 \pm 19$; DM2 plus cholesterol, 292 \pm 50 ; DM2 plus ergosterol, $120 \pm 23$; DM2 plus cholestane, $73 \pm 5$; and DM2 plus squalene, $56 \pm 12$, all of which are significantly different from one another $(P<0 \cdot 05)$. Although cholesterol-supplemented mycelium produced almost twice as much phospholipid as that grown on defined medium, the other sterol which induces sporulation, ergosterol, supported growth of mycelium with less phospholipid than that with no sterol supplement. Attempts to assess the effect of testosterone supplementation were unsuccessful because this steroid inhibited fungal growth.

Recently we demonstrated that electrospray MS and MS/MS can be used to identify glycerophospholipid molecular species with minimal sample preparation (Figs 1-3). With proper adjustment of operating parameters, over $90 \%$ of signal intensity in a typical positive ion spectrum was due to $[M+\mathrm{H}]^{+}$(or $[M+60]^{+}$for cholinecontaining species) ions. For diacyl species, this technique does not identify which fatty acids are found in the $s n-1$ or $s n-2$ positions. We follow the accepted practice of reporting the less unsaturated fatty acid as occurring in the $s n-1$ position. Another limitation involves isobaric ether lipids. As an example, it is not possible without subclass separation to differentiate between a mono- 


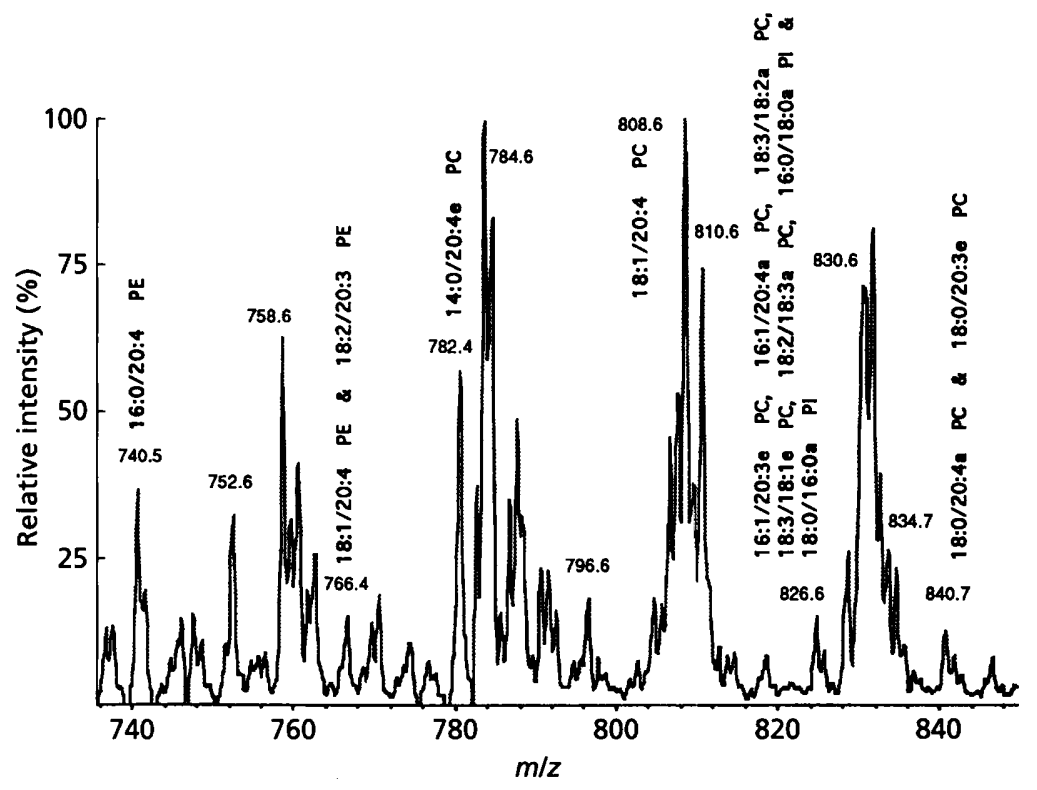

Fig. 1. Positive electrospray mass spectrum from $\mathrm{m} / \mathrm{z} \quad 735$ to $\mathrm{m} / \mathrm{z} \quad 850$ of glycerophospholipids from $L$. giganteum nuclei isolated from mycelium grown with a cholestane supplement. Representative peaks are marked, and six are labelled with the corresponding component molecular species that were identified using positive and negative ion electrospray $\mathrm{MS} / \mathrm{MS}$. $\mathrm{PE}$, glycerophosphoethanolamine; PC, glycerophosphocholine; PI, glycerophosphoinositol; a, alkyl-acyl species; e, alkenyl-acyl species; all others are diacyl compounds. Peaks correspond to $[M+H]^{+}$ions except for ethercontaining glycerophosphocholine species, which are found either as $[M+60]^{+}$or $[M+44]^{+}$due to acetate adduct formation with or without loss of $-\mathrm{CH}_{3}$ from the phosphocholine headgroup.

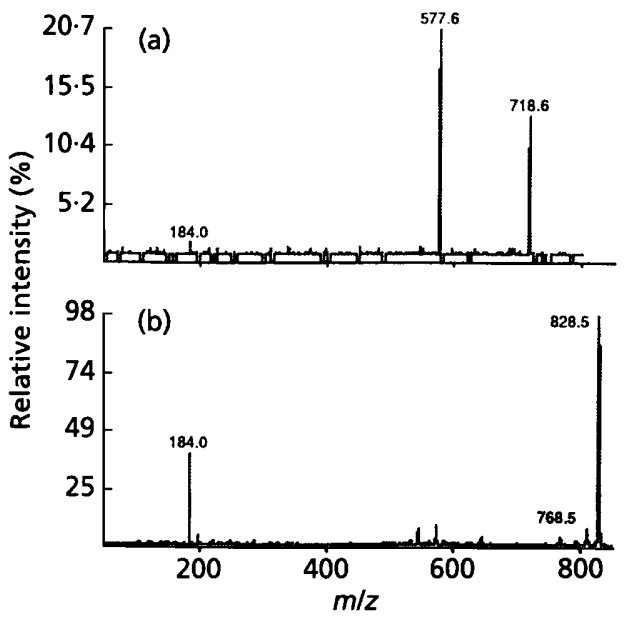

Fig. 2. (a) Positive ion electrospray MS/MS of $\mathrm{m} / \mathrm{z} 718$, produced by 16:0/18:1 phosphatidylethanolamine and a small amount of 14:0/18:0 alkenyl-acyl glycerophosphocholine. Loss of phosphoethanolamine $(-141)$ from $\mathrm{m} / \mathrm{z} 718$ produces the peak at $\mathrm{m} / \mathrm{z}$ 577. (b) Positive ion electrospray MS/MS of $\mathrm{m} / \mathrm{z} 828$, the acetate adduct of $\mathrm{m} / \mathrm{z} 768$, corresponding to $M^{+}$of 16:0/20:4 alkyl-acyl glycerophosphocholine. The phosphocholine headgroup produces a well-defined peak at $\mathrm{m} / \mathrm{z} 184$.

unsaturated 18-carbon alkyl-linked moiety and a saturated 18-carbon alkenyl-linked species. Species reported in this paper are considered the most likely combinations to be synthesized based on fatty acid profiles of the L. giganteum phospholipid fraction. For instance, the fungus produces very small amounts of myristoleic acid $(14: 1 \omega 5)$, so it is unlikely that the glycerophosphocholine species reported as 14:0/20:4 alkenyl-acyl, which makes up a significant percentage of this lipid class (see Table 2), is actually a $14: 1 / 20: 4$ alkyl-acyl species. Interpretation of spectra for this fungus is somewhat simplified since it does not synthesize $18: 4,20: 5$ or any 22 -carbon fatty acids.

Scanning over a mass range of $m / z 450$ or 600 to $\mathrm{m} / \mathrm{z}$ 1000 , relative peak intensities for molecular species of GPC, GPI, GPE and monomethylphosphatidylethanolamine for L. giganteum mycelium and nuclei were as reproducible as those found for analyses of commercially available glycerophospholipids (Kerwin $e t$ al., 1994; data not shown). This served as a check for the reliability of using electrospray MS and MS/MS to characterize $L$. giganteum glycerophospholipid composition. Three classes of glycerophospholipid were chosen for detailed analysis: GPC and GPE, which together comprise a minimum of $70 \%$ of total cell glycerophospholipid, and GPI, which has a pivotal role in regulation of cellular processes in many eukaryotic cells. The most surprising result was the almost unprecedented high levels of ether lipids found in GPC and GPI, with less, but still appreciable quantities, in GPE (Table 1). This could be an artefact of electrospray MS, but there was no evidence of preferential ionization of ether lipids in commercially available glycerophospholipids, all of which had been previously analysed using other methods (Kerwin et al., 1994). This included a preparation of bovine brain GPE which contained approximately $60 \%$ ether lipids.

There were significant differences in relative molecular species composition in all three classes of glycerophospholipid from mycelium grown on the different media (data not shown). Many of these were minor components, but others such as the 14:0/20:4 ether GPC, $18: 3 / 20: 4$ alkenyl-acyl GPC and $18: 1 / 20: 4$ and $18: 2 / 20: 4$ diacyl GPC made up a significant percentage of their lipid class. Of the three lipid classes, the GPE molecular species varied the least among mycelia cultured on the different growth media, although there was one 


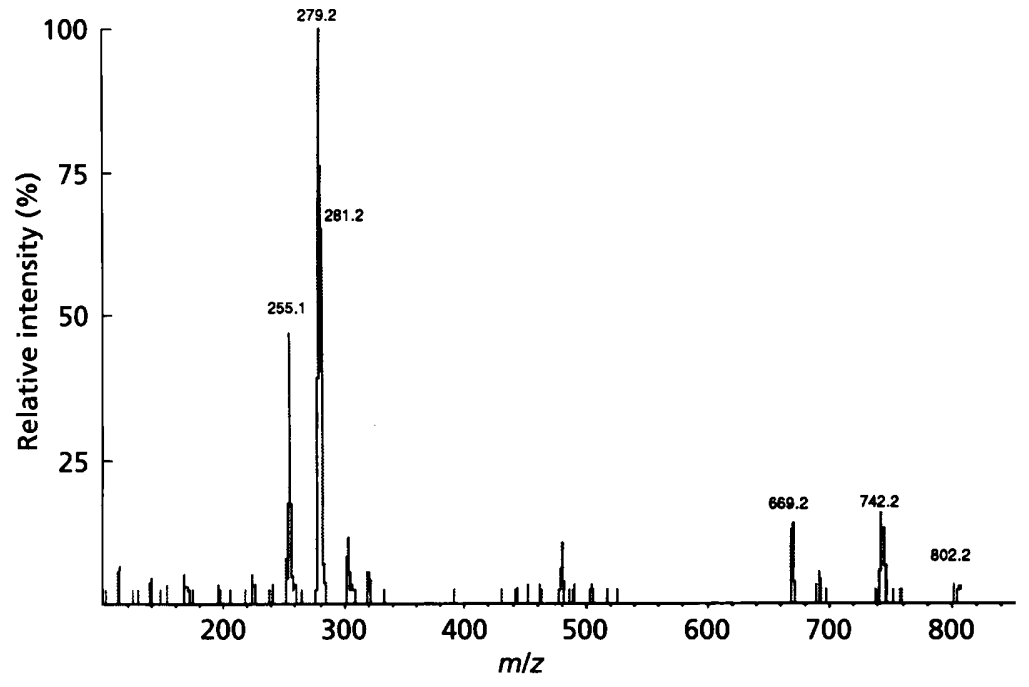

\begin{abstract}
Fig. 3. Negative ion electrospray MS/MS of $\mathrm{m} / \mathrm{z} 802$, the acetate adduct of $\mathrm{m} / \mathrm{z} 742$. Peaks at $\mathrm{m} / \mathrm{z} 255,279$ and 281 corresponding to RCOO- of 16:0, 18:2 and $18: 1$, respectively, confirm that the peak at $\mathrm{m} / \mathrm{z} 742$ corresponds to three ether-linked glycerophosphocholine moieties: 18:3/16:0 alkyl-acyl, 16:1/18:2 alkyl-acyl and 16:1/18:1 alkenyl-acyl.
\end{abstract}

Table 1. Percentage ether glycerophospholipids in three lipid classes from L. giganteum grown on defined media

Values are $(\mathrm{mol} \%)$ mean \pm SD from three separate cultures; figures in a given row with matching superscript letters are significantly different from one another $(P<0 \cdot 05)$. Values for nuclear preparations followed by an asterisk are significantly different in percentage composition from those found in total cell phospholipids. Data were generated by dividing peak intensities corresponding to alkyl-acyl plus alkenyl-acyl moieties by total peak intensity for a given lipid class. DM2, defined medium; DM2CHO, defined medium + cholesterol; DM2ERG, defined medium + ergosterol; DM2CHN, defined medium + cholestane; DM2SQN, defined medium + squalene.

\begin{tabular}{|c|c|c|c|c|c|}
\hline \multirow[t]{2}{*}{ Lipid class } & \multicolumn{5}{|c|}{ Percentage composition after growth on: } \\
\hline & DM2 & DM2CHO & DM2ERG & DM2CHN & DM2SQN \\
\hline \multicolumn{6}{|l|}{ Total cell glycerophospholipid } \\
\hline Glycerophosphocholine & $74 \cdot 0 \pm 2 \cdot 65^{a}$ & $74 \cdot 3 \pm 2 \cdot 1^{b}$ & $80 \cdot 0 \pm 2 \cdot 0^{c, d}$ & $69 \pm 1 \cdot 7^{a, b, c}$ & $73 \cdot 0 \pm 1 \cdot 7^{d}$ \\
\hline Glycerophosphoethanolamine & $12 \cdot 6 \pm 2 \cdot 6^{a, b}$ & $19 \cdot 8 \pm 3 \cdot 6^{a, c}$ & $16 \cdot 8 \pm 1 \cdot 9^{b, d}$ & $13 \cdot 0 \pm 1 \cdot 2^{c, d, e}$ & $15 \cdot 4 \pm 1 \cdot 1^{e}$ \\
\hline Glycerophosphocholine & $71 \cdot 3 \pm 3 \cdot 1^{a}$ & $58 \cdot 3 \pm 2 \cdot 1 * a, b, c, d$ & $76 \pm 5 \cdot 2^{b}$ & $78 \cdot 0 \pm 1 \cdot 0 * c$ & $81 \cdot 3 \pm 2 \cdot 1^{* a, d}$ \\
\hline Glycerophosphoethanolamine & $10 \cdot 3 \pm 2 \cdot 1^{a}$ & $12 \cdot 7 \pm 3 \cdot 8$ & $15 \cdot 7 \pm 4 \cdot 0$ & $7 \cdot 3 \pm 2 \cdot 1 * a$ & $12 \cdot 3 \pm 2 \cdot 1$ \\
\hline Glycerophosphoinositol & $81 \cdot 7 \pm 0 \cdot 6^{a . b}$ & $84 \cdot 7 \pm 5 \cdot 1$ & $79 \cdot 3 \pm 2 \cdot 5^{c}$ & $86 \cdot 7 \pm 1 \cdot 5^{* a, c, d}$ & $77 \cdot 3 \pm 0 \cdot 6^{b, d}$ \\
\hline
\end{tabular}

significant difference as discussed below. Some of the molecular species were unusual in having polyunsaturated

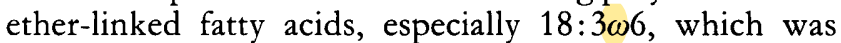
found in alkyl-acyl and alkenyl-acyl species of GPC (Table 2), GPE (Table 3) and GPI (Table 4) in both whole cell and nuclear fractions.

We were most interested in differences in molecular species produced by $L$. giganteum grown on media supplemented with cholesterol and ergosterol, which induce sporulation, relative to the other three media. The one consistent difference observed when comparing mycelium grown on ergosterol and cholesterol with that grown on other media was an elevated percentage of total ether lipids in the GPE fraction compared to mycelium from cultures grown on defined medium alone or on defined medium plus cholestane (Table 1).

\section{Nuclear glycerophospholipids}

Initial attempts to isolate $L$. giganteum nuclei followed the standard protocol of incubation with lytic enzymes in order to obtain protoplasts, which could subsequently be gently disrupted as the first step in obtaining these organelles. Unexpectedly, the cell walls of this fungus proved to be resistant to degradation by a wide variety of enzymic treatments with or without various preincubations or subsequent osmotic shock. Enzymes which proved to be ineffective either singly or in various combinations included three proteases, glusulase, laminarinase, three cellulases, Novozym 234, $\beta$ glucuronidase, pectinase, driselase, chitinase, lyticase, macerozyme, zymolase and lyticase. After trying a number of alternatives, it was found that a combination of chilling in liquid nitrogen and mechanical abrasion would break 
Table 2. Glycerophosphocholine molecular species from nuclei of $L$. giganteum grown on defined media

Values are $(\mathrm{mol} \%)$ mean $\pm S D$ from three separate cultures; figures in a given row with matching superscript letters are significantly different from one another $(P<0.05)$. Values followed by an asterisk are significantly different in percentage composition from those found in total cell phospholipids. Alk, alkyl-acyl molecular species; enyl, alkenyl-acyl species; all others are diacyl species. DM2, defined medium; DM2CHO, defined medium + cholesterol; DM2ERG, defined medium + ergosterol; DM2CHN, DM2+ cholestane; DM2SQN, $\mathrm{DM} 2+$ squalene.

\begin{tabular}{|c|c|c|c|c|c|}
\hline \multirow{2}{*}{$\begin{array}{l}\text { Molecular } \\
\text { species }\end{array}$} & \multicolumn{5}{|c|}{ Percentage composition after growth on: } \\
\hline & DM2 & DM2CHO & DM2ERG & $\mathbf{D M} 2 \mathrm{CHN}+$ & DM2SQN \\
\hline 14:0/18:1 alk & $0 \cdot 38 \pm 0 \cdot 02^{a}$ & $0.62 \pm 0.31 *$ & $0.95 \pm 0.09^{a}$ & $0^{* a, b}$ & $0 \cdot 86 \pm 0 \cdot 25^{b}$ \\
\hline $14: 0 / 18: 0$ alk & $2 \cdot 80 \pm 0 \cdot 12^{a}$ & $3.95 \pm 0.65^{*}$ & $1.98 \pm 0 \cdot 40^{* b}$ & $5 \cdot 00 \pm 0 \cdot 20^{* a, b}$ & $2.95 \pm 1.07$ \\
\hline $16: 0 / 16: 0$ & $0 \cdot 22 \pm 0 \cdot 07$ & $0 \cdot 26 \pm 0 \cdot 11$ & $0.21 \pm 0.08$ & $0 \cdot 32 \pm 0 \cdot 08^{a}$ & $0 \cdot 25 \pm 0 \cdot 08^{a}$ \\
\hline $14: 0 / 20: 4$ enyl & $14 \cdot 62 \pm 2 \cdot 02 *$ & $16.98 \pm 5.49$ & $17 \cdot 88 \pm 2 \cdot 50^{a}$ & $14 \cdot 52 \pm 2 \cdot 89$ & $23 \cdot 23 \pm 1 \cdot 91^{a}$ \\
\hline $14: 0 / 20: 4$ alk & $4 \cdot 48 \pm 0.90$ & $9 \cdot 87 \pm 1 \cdot 68^{a, b}$ & $6.76 \pm 2.48$ & $5.94 \pm 1.55^{a}$ & $4 \cdot 80 \pm 1 \cdot 37^{b}$ \\
\hline $16: 0 / 18: 1$ enyl & $0 \cdot 25 \pm 0 \cdot 04^{* a}$ & $0 \cdot 22 \pm 0 \cdot 12^{c}$ & $0 \cdot 25 \pm 0 \cdot 16^{b}$ & $0 \cdot 72 \pm 0 \cdot 18^{a, b}$ & $0 \cdot 57 \pm 0 \cdot 24^{c}$ \\
\hline $16: 0 / 18: 2$ alk & $0 \cdot 34 \pm 0 \cdot 06^{a}$ & $0 \cdot 29 \pm 0 \cdot 15^{b}$ & $0 \cdot 33 \pm 0 \cdot 21$ & $0^{* a}$ & $0 \cdot 75 \pm 0 \cdot 31^{b}$ \\
\hline $18: 1 / 16: 0$ enyl & $0 \cdot 08 \pm 0.01^{a}$ & $0 \cdot 07 \pm 0 \cdot 04^{b}$ & $0.08 \pm 0.05$ & $0^{* a, c}$ & $0 \cdot 26 \pm 0 \cdot 18^{b, c}$ \\
\hline $16: 0 / 18: 1$ alk & $0 \cdot 21 \pm 0 \cdot 09^{a}$ & $0 \cdot 28 \pm 0 \cdot 30$ & $0 \cdot 16 \pm 0.09^{a}$ & $0 \cdot 67 \pm 0.24^{a}$ & $0 \cdot 31 \pm 0 \cdot 05$ \\
\hline $18: 0 / 16: 1 \mathrm{alk}$ & $0 \cdot 18 \pm 0 \cdot 08^{a}$ & $0.25 \pm 0.26$ & $0 \cdot 13 \pm 0 \cdot 08^{a}$ & $0 * b$ & $0 \cdot 27 \pm 0.05^{b}$ \\
\hline $18: 1 / 16: 0$ alk & $0.09 \pm 0.04^{a}$ & $0 \cdot 12 \pm 0 \cdot 13$ & $0 \cdot 11 \pm 0.06$ & $0^{* a, b}$ & $0 \cdot 13 \pm 0.02^{b}$ \\
\hline $18: 0 / 16: 0$ alk & $0 \cdot 08 \pm 0 \cdot 04^{a}$ & $0.06 \pm 0.02^{b}$ & $0.08 \pm 0.05^{c}$ & $9 \cdot 87 \pm 3 \cdot 29^{* a, b, c, d}$ & $0 \cdot 15 \pm 0.05^{d}$ \\
\hline $16: 1 / 20: 3$ enyl & $0 \cdot 17 \pm 0 \cdot 02 * a$ & $0 \cdot 18 \pm 0 \cdot 06^{b}$ & $0 \cdot 13 \pm 0 \cdot 08$ & $0 \cdot 22 \pm 0 \cdot 03^{*}$ & $0 \cdot 27 \pm 0 \cdot 06^{* a, b}$ \\
\hline $16: 1 / 20: 4$ alk & $0 \cdot 76 \pm 0 \cdot 12^{a}$ & $0 \cdot 80 \pm 0 \cdot 25^{b}$ & $0.60 \pm 0.34$ & $0.99 \pm 0.13^{*}$ & $1 \cdot 25 \pm 0 \cdot 26^{* a, b}$ \\
\hline $18: 3 / 18: 2 \mathrm{alk}$ & $0.23 \pm 0.04^{a}$ & $0 \cdot 24 \pm 0 \cdot 08$ & $0 \cdot 19 \pm 0 \cdot 11$ & $0 \cdot 30 \pm 0.04^{*}$ & $0.38 \pm 0.08^{* a}$ \\
\hline $18: 3 / 18: 1$ enyl & $0.22 \pm 0.03^{* a}$ & $0.23 \pm 0.07^{b}$ & $0 \cdot 18 \pm 0 \cdot 10$ & $0.29 \pm 0.03^{*}$ & $0.36 \pm 0.07^{* a, b}$ \\
\hline $18: 0 / 18: 3$ enyl & $0 \cdot 89 \pm 0 \cdot 16^{* a}$ & $0 \cdot 49 \pm 0 \cdot 21$ & $0 \cdot 43 \pm 0 \cdot 21$ & $0.25 \pm 0.02^{* a}$ & $1.65 \pm 0.66$ \\
\hline $18: 1 / 18: 2$ enyl & $2 \cdot 07 \pm 0.37^{* a}$ & $1 \cdot 15 \pm 0 \cdot 48$ & $1.00 \pm 0.50$ & $0^{* a, b}$ & $3 \cdot 85 \pm 1 \cdot 55^{b}$ \\
\hline $18: 0 / 18: 2$ enyl & $9 \cdot 34 \pm 0.53 * a, c$ & $0.38 \pm 0.06^{* a, b}$ & $8 \cdot 46 \pm 1 \cdot 54^{b}$ & $0^{* b, c}$ & $7 \cdot 04 \pm 0 \cdot 74^{a, c}$ \\
\hline $18: 1 / 18: 1$ enyl & $6.60 \pm 0.37 * a, b$ & $0 \cdot 27 \pm 0 \cdot 04^{* a, b}$ & $6 \cdot 06 \pm 1 \cdot 14^{b}$ & $3 \cdot 74 \pm 1 \cdot 30^{b}$ & $4.97 \pm 0.53^{a}$ \\
\hline $20: 3 / 16: 0$ alk & $4 \cdot 12 \pm 0 \cdot 23^{* a}$ & $0 \cdot 17 \pm 0 \cdot 03^{* a, b, c}$ & $3 \cdot 73 \pm 0 \cdot 68^{b, c}$ & $1 \cdot 02 \pm 0.36^{* a, c}$ & $3 \cdot 11 \pm 0 \cdot 33^{a}$ \\
\hline $18: 0 / 18: 2 \mathrm{alk}$ & $2 \cdot 63 \pm 0 \cdot 26^{a . b}$ & $0 \cdot 27 \pm 0 \cdot 16^{* a, d}$ & $3 \cdot 24 \pm 1 \cdot 23^{c}$ & $0^{* b, c, e}$ & $2 \cdot 36 \pm 0 \cdot 77^{d, e}$ \\
\hline $18: 1 / 18: 1$ alk & $2 \cdot 07 \pm 0 \cdot 21^{a, b}$ & $0 \cdot 21 \pm 0 \cdot 13^{* a, d}$ & $2.54 \pm 0.97^{c}$ & $0^{* b, c, e}$ & $1 \cdot 85 \pm 0 \cdot 60^{d, e}$ \\
\hline $18: 3 / 20: 4$ enyl & $7 \cdot 41 \pm 1 \cdot 07^{a, c}$ & $3 \cdot 59 \pm 0 \cdot 23^{* a, b}$ & $9 \cdot 49 \pm 1 \cdot 66^{b, d}$ & $2 \cdot 24 \pm 0 \cdot 55^{* c, d . e}$ & $6 \cdot 10 \pm 1 \cdot 37^{* e}$ \\
\hline $18: 3 / 20: 4$ alk & $2 \cdot 10 \pm 0 \cdot 81$ & $1 \cdot 78 \pm 0.58^{a}$ & $2 \cdot 97 \pm 1 \cdot 28$ & $0 \cdot 33 \pm 0 \cdot 17^{* a, b}$ & $2 \cdot 06 \pm 0.66^{b}$ \\
\hline $18: 2 / 20: 4$ alk & $1 \cdot 22 \pm 0 \cdot 25^{a}$ & $3.03 \pm 0 \cdot 81^{* b}$ & $1 \cdot 71 \pm 0.63^{c}$ & $8 \cdot 21 \pm 0 \cdot 39 * a, b, c, d$ & $2 \cdot 08 \pm 0 \cdot 13^{* d}$ \\
\hline $20: 3 / 18: 2$ alk & $0.36 \pm 0.22^{a}$ & $0.56 \pm 0.13^{* b}$ & $0 \cdot 22 \pm 0 \cdot 06^{b, c}$ & $1 \cdot 19 \pm 0 \cdot 06^{* a, b}$ & $1 \cdot 12 \pm 0 \cdot 31^{* c}$ \\
\hline $18: 1 / 20: 4$ alk & $0.58 \pm 0 \cdot 11^{a, c}$ & $1 \cdot 46 \pm 0.35^{* a . b}$ & $0 \cdot 57 \pm 0 \cdot 15^{b}$ & $4 \cdot 72 \pm 0 \cdot 68^{* a, b, c}$ & $0 \cdot 80 \pm 0 \cdot 10^{* c}$ \\
\hline $18: 1 / 20: 3$ enyl & $0 \cdot 25 \pm 0.05^{a, c}$ & $0 \cdot 64 \pm 0 \cdot 15^{* a, b}$ & $0.25 \pm 0.07^{b}$ & $0.36 \pm 0.02$ & $0.35 \pm 0.04^{* c}$ \\
\hline $18: 0 / 20: 4$ alk & $1 \cdot 09 \pm 0.29^{a, b}$ & $2 \cdot 34 \pm 0.73^{a}$ & $1.09 \pm 0.50$ & $1 \cdot 96 \pm 0 \cdot 28$ & $0.96 \pm 0.73^{b}$ \\
\hline $18: 0 / 20: 3$ enyl & $0 \cdot 12 \pm 0 \cdot 03$ & $0 \cdot 26 \pm 0 \cdot 08$ & $0 \cdot 12 \pm 0 \cdot 05$ & $0.12 \pm 0.02$ & $0.16 \pm 0.03 *$ \\
\hline $18: 3 / 16: 0$ alk & $0 \cdot 17 \pm 0 \cdot 03^{a}$ & $0 \cdot 12 \pm 0 \cdot 04^{b}$ & $0 \cdot 12 \pm 0 \cdot 08^{c}$ & $1 \cdot 26 \pm 0 \cdot 10^{* a, b, c, d}$ & $0 \cdot 16 \pm 0 \cdot 03^{* d}$ \\
\hline $16: 1 / 18: 2 \mathrm{alk}$ & $0.35 \pm 0.07^{a}$ & $0 \cdot 26 \pm 0 \cdot 08^{a, b}$ & $0 \cdot 25 \pm 0 \cdot 17$ & $0^{a, b}$ & $0.35 \pm 0.08^{* b}$ \\
\hline $16: 1 / 18: 1$ alk & $0 \cdot 52 \pm 0 \cdot 10^{a}$ & $0 \cdot 39 \pm 0 \cdot 12^{a, b}$ & $0 \cdot 36 \pm 0 \cdot 25$ & $0^{a, b}$ & $0 \cdot 51 \pm 0 \cdot 11^{* b}$ \\
\hline $18: 3 / 20: 4$ & $5 \cdot 29 \pm 0 \cdot 53^{a, b}$ & $6 \cdot 32 \pm 4.65$ & $2 \cdot 84 \pm 0.78^{a}$ & $3 \cdot 27 \pm 0.73^{b}$ & $4 \cdot 17 \pm 0 \cdot 46$ \\
\hline $18: 2 / 20: 4$ & $9 \cdot 62 \pm 2 \cdot 50^{*}$ & $11 \cdot 78 \pm 1 \cdot 30^{* a . b, c}$ & $6.28 \pm 0.74^{a}$ & $7 \cdot 79 \pm 1 \cdot 25^{* b}$ & $6.08 \pm 0.78^{* c}$ \\
\hline $18: 1 / 20: 4$ & $6 \cdot 71 \pm 1 \cdot 42^{a}$ & $11 \cdot 17 \pm 2 \cdot 25^{b}$ & $6 \cdot 48 \pm 2 \cdot 45$ & $0 \cdot 99 \pm 0.25^{* a, b}$ & $3.67 \pm 0.83^{* a, b}$ \\
\hline $18: 2 / 20: 3$ & $2 \cdot 36 \pm 0 \cdot 49^{a}$ & $3.92 \pm 0.79^{b}$ & $2 \cdot 28 \pm 0 \cdot 86^{c}$ & $0^{* a, b, c}$ & $1 \cdot 29 \pm 0 \cdot 30^{* a, b}$ \\
\hline $18: 0 / 20: 4$ & $4 \cdot 29 \pm 1 \cdot 50^{a}$ & $8 \cdot 10 \pm 0 \cdot 92^{b}$ & $6 \cdot 10 \pm 0.93^{* c}$ & $9 \cdot 55 \pm 1 \cdot 11^{a, c, d}$ & $3 \cdot 31 \pm 0.37^{b, d}$ \\
\hline $20: 3 / 20: 4$ alk & $0.97 \pm 0 \cdot 22^{a}$ & $1 \cdot 63 \pm 0.81$ & $0.81 \pm 0.39$ & $0^{* a, b}$ & $1 \cdot 13 \pm 0 \cdot 19^{* b}$ \\
\hline $20: 0 / 20: 4$ enyl & $1 \cdot 85 \pm 0.52^{a}$ & $2.53 \pm 0.06^{* b}$ & $1 \cdot 39 \pm 0.66$ & $0 \cdot 78 \pm 0 \cdot 11^{a, b}$ & $1.75 \pm 0.43 *$ \\
\hline $20: 0 / 20: 4$ alk & $1 \cdot 68 \pm 0 \cdot 45$ & $2 \cdot 42 \pm 0 \cdot 81$ & $1 \cdot 08 \pm 0.64$ & $0.57 \pm 0.07 * a$ & $1.57 \pm 0.09 * a$ \\
\hline $20: 0 / 20: 3$ enyl & $0.22 \pm 0.06$ & $0 \cdot 32 \pm 0 \cdot 11$ & $0.14 \pm 0.09$ & $0.17 \pm 0.02$ & $0 \cdot 21 \pm 0 \cdot 01^{*}$ \\
\hline
\end{tabular}

† Other molecular species identified in nuclei from cholestane-supplemented media were: $16: 1 / 18: 0$ alkenyl acyl, $0 \cdot 60 \pm 0 \cdot 15 \% ; 18: 2 / 18: 3$ alkenyl-acyl, $1 \cdot 60 \pm 0 \cdot 19 \% ; 18: 3 / 18: 2$ alkenyl-acyl, $2 \cdot 28 \pm 0 \cdot 27 \% ; 16: 1 / 20: 4$ alkenyl-acyl, $0 \cdot 57 \pm 0 \cdot 07 \% ; 18: 3 / 18: 1$ alkyl-acyl, $0 \cdot 41 \pm 0.03 \% ; 18: 3 / 16: 0$ alkyl-acyl, $6 \cdot 57 \pm 2 \cdot 29 \% ; 18: 2 / 20: 4$ alkenyl-acyl, $0 \cdot 58 \pm 0 \cdot 29 \%$. 
Table 3. Glycerophosphoethanolamine molecular species from nuclei of L. giganteum grown on defined media

Values are $(\mathrm{mol} \%)$ mean \pm sD from three separate cultures; figures in a given row with matching superscript letters are significantly different from one another $(P<0 \cdot 05)$. Values followed by an asterisk are significantly different in percentage composition from those found in total cell phospholipids. Alk, alkyl-acyl molecular species; enyl, alkenyl-acyl species; all others are diacyl species. DM2, defined medium; DM2CHO, defined medium + cholesterol; DM2ERG, defined medium + ergosterol; DM2CHN, DM2+ cholestane; DM2SQN, DM2 + squalene.

\begin{tabular}{|c|c|c|c|c|c|}
\hline \multirow{2}{*}{$\begin{array}{l}\text { Molecular } \\
\text { species }\end{array}$} & \multicolumn{5}{|c|}{ Percentage composition after growth on: } \\
\hline & DM2 & DM2CHO & DM2ERG & DM2CHN $\dagger$ & DM2SQN \\
\hline $14: 0 / 18: 2$ & $1 \cdot 49 \pm 0 \cdot 61^{a, b, c}$ & $2 \cdot 02 \pm 0 \cdot 79^{a}$ & $1.68 \pm 0.62$ & $3 \cdot 17 \pm 0 \cdot 20^{* b}$ & $1 \cdot 32 \pm 0 \cdot 37^{c}$ \\
\hline $14: 0 / 18: 1$ & $1 \cdot 24 \pm 0 \cdot 11^{*}$ & $3.61 \pm 1.07$ & $1.75 \pm 0.77$ & $5 \cdot 62 \pm 1 \cdot 84$ & $2 \cdot 39 \pm 0.48$ \\
\hline $16: 1 / 16: 1$ & $0.80 \pm 0.33^{a}$ & $1 \cdot 09 \pm 0.43$ & $0 \cdot 91 \pm 0 \cdot 33$ & $0 \cdot 79 \pm 0 \cdot 05^{a}$ & $0 \cdot 71 \pm 0 \cdot 20$ \\
\hline $16: 0 / 16: 1$ & $2 \cdot 42 \pm 0 \cdot 21^{a, b}$ & $7 \cdot 01 \pm 2 \cdot 08$ & $3 \cdot 40 \pm 1 \cdot 49$ & $10 \cdot 71 \pm 3 \cdot 79^{a}$ & $4 \cdot 63 \pm 0 \cdot 91^{a}$ \\
\hline $16: 0 / 16: 0$ & $5 \cdot 53 \pm 2 \cdot 13$ & $6 \cdot 46 \pm 3 \cdot 21^{a}$ & $5 \cdot 42 \pm 1 \cdot 04$ & $7 \cdot 22 \pm 2 \cdot 09$ & $9 \cdot 91 \pm 3 \cdot 71^{a}$ \\
\hline $18: 3 / 16: 0$ enyl & $4 \cdot 20 \pm 0 \cdot 92$ & $5 \cdot 26 \pm 2 \cdot 00^{* a}$ & $9 \cdot 12 \pm 3 \cdot 09^{a}$ & $2 \cdot 38 \pm 0 \cdot 61 *$ & $5 \cdot 13 \pm 1 \cdot 43$ \\
\hline $16: 1 / 18: 2$ enyl & $2 \cdot 36 \pm 0.52^{a}$ & $2 \cdot 96 \pm 1 \cdot 12^{* b}$ & $5 \cdot 13 \pm 1 \cdot 74^{b}$ & $1 \cdot 14 \pm 0 \cdot 30^{* a}$ & $2 \cdot 89 \pm 0 \cdot 80$ \\
\hline $14: 0 / 20: 4$ & $0.67 \pm 0 \cdot 10$ & $1.27 \pm 0.69^{a}$ & $0.84 \pm 0.22$ & $2 \cdot 03 \pm 0.58^{a}$ & $0.63 \pm 0.24$ \\
\hline $16: 1 / 18: 3$ & $0 \cdot 36 \pm 0.06^{a}$ & $0 \cdot 68 \pm 0.37^{b}$ & $0 \cdot 45 \pm 0 \cdot 12$ & $0 * a, b$ & $0 \cdot 34 \pm 0 \cdot 13$ \\
\hline $16: 0 / 18: 2$ & $1 \cdot 38 \pm 0 \cdot 28$ & $1 \cdot 30 \pm 0.33$ & $1 \cdot 25 \pm 0.06$ & $2 \cdot 39 \pm 0.50$ & $1 \cdot 56 \pm 0 \cdot 23$ \\
\hline $16: 0 / 18: 1$ & $3 \cdot 72 \pm 0 \cdot 14^{a}$ & $7 \cdot 56 \pm 1 \cdot 19^{* a}$ & $13 \cdot 51 \pm 4 \cdot 71$ & $5 \cdot 11 \pm 2 \cdot 05$ & $5 \cdot 29 \pm 0 \cdot 44$ \\
\hline $16: 0 / 18: 0$ & $0.75 \pm 0.04^{a, b}$ & $1 \cdot 95 \pm 0 \cdot 17^{* a, c}$ & $2.35 \pm 1.09$ & $10 \cdot 46 \pm 3 \cdot 33^{* b, c, d}$ & $1.49 \pm 0.67^{d}$ \\
\hline $16: 0 / 20: 4$ & $5 \cdot 13 \pm 0 \cdot 79 * a$ & $7 \cdot 73 \pm 2 \cdot 87^{b}$ & $6 \cdot 72 \pm 3 \cdot 17^{c}$ & $33 \cdot 82 \pm 1 \cdot 86^{* a, b, c, d}$ & $5 \cdot 80 \pm 0 \cdot 65^{* d}$ \\
\hline $16: 0 / 20: 3$ & $1 \cdot 96 \pm 0.54^{a}$ & $1 \cdot 75 \pm 0 \cdot 32^{b}$ & $1.89 \pm 0.42^{c}$ & $0 \cdot 23 \pm 0 \cdot 05^{* a, b, c}$ & $2 \cdot 47 \pm 0 \cdot 44^{b}$ \\
\hline $18: 0 / 18: 2$ & $1.65 \pm 0.35$ & $1 \cdot 87 \pm 0 \cdot 30^{a}$ & $2 \cdot 11 \pm 0 \cdot 52^{b}$ & $0^{* a, b, c}$ & $2 \cdot 20 \pm 0 \cdot 34^{c}$ \\
\hline $18: 3 / 20: 4$ enyl & $0 \cdot 45 \pm 0 \cdot 21$ & $0 \cdot 49 \pm 0 \cdot 14^{a}$ & $0 \cdot 43 \pm 0 \cdot 10^{b}$ & $0^{* a, b, c}$ & $0 \cdot 42 \pm 0 \cdot 11^{c}$ \\
\hline $18: 2 / 20: 4$ enyl & $0 \cdot 38 \pm 0 \cdot 17$ & $0 \cdot 30 \pm 0.27$ & $0.43 \pm 0.17$ & $1.51 \pm 0.99$ & $0 \cdot 44 \pm 0 \cdot 13$ \\
\hline $18: 2 / 20: 4$ alk & $2 \cdot 87 \pm 0 \cdot 24^{a}$ & $3 \cdot 37 \pm 0 \cdot 50^{b}$ & $3.81 \pm 1.57$ & $1.42 \pm 0.43^{* a, b, c}$ & $3 \cdot 44 \pm 0 \cdot 15^{c}$ \\
\hline $18: 3 / 20: 4$ & $2 \cdot 99 \pm 0 \cdot 30$ & $4 \cdot 78 \pm 1 \cdot 02$ & $6 \cdot 91 \pm 3 \cdot 30$ & $2 \cdot 17 \pm 1 \cdot 51$ & $2 \cdot 20 \pm 0 \cdot 50$ \\
\hline $18: 2 / 20: 4$ & $10 \cdot 48 \pm 0 \cdot 80^{* a}$ & $7 \cdot 11 \pm 0 \cdot 89^{a}$ & $7 \cdot 76 \pm 1 \cdot 38^{b}$ & $0 \cdot 92 \pm 0 \cdot 44^{* a, b, c}$ & $7 \cdot 59 \pm 1 \cdot 00^{c}$ \\
\hline $18: 1 / 20: 4$ & $10 \cdot 14 \pm 0 \cdot 56^{a}$ & $12 \cdot 29 \pm 2 \cdot 53^{b}$ & $9 \cdot 34 \pm 0 \cdot 82^{c}$ & $1 \cdot 21 \pm 0 \cdot 77^{* a, b, c}$ & $10 \cdot 51 \pm 0 \cdot 92^{c}$ \\
\hline $18: 2 / 20: 3$ & $3 \cdot 38 \pm 0 \cdot 19^{a}$ & $4 \cdot 09 \pm 0 \cdot 84^{b}$ & $3 \cdot 11 \pm 0 \cdot 27^{c}$ & $0^{* a, b, c}$ & $3 \cdot 50 \pm 0 \cdot 31^{c}$ \\
\hline $18: 0 / 20: 4$ & $4 \cdot 30 \pm 0 \cdot 50^{a}$ & $3.97 \pm 1.33^{b}$ & $2 \cdot 68 \pm 0 \cdot 56^{a}$ & $0^{* a, b}$ & $4 \cdot 79 \pm 0.82$ \\
\hline $18: 1 / 20: 3$ & $4.92 \pm 0.56^{a}$ & $4.53 \pm 1.52$ & $3 \cdot 07 \pm 0 \cdot 64^{a}$ & $1 \cdot 15 \pm 0 \cdot 88^{b}$ & $5.48 \pm 0.94^{b}$ \\
\hline $20: 3 / 20: 3$ & $26 \cdot 40 \pm 1 \cdot 15^{* a, b}$ & $6.55 \pm 2.82^{a}$ & $5 \cdot 14 \pm 1 \cdot 83^{b}$ & $0^{* b}$ & $14 \cdot 86 \pm 6 \cdot 17$ \\
\hline
\end{tabular}

†Other molecular species identified in nuclei from cholestane-supplemented media were: $16: 1 / 20: 3$ diacyl, $5 \cdot 60 \pm 0 \cdot 26 \% ; 18: 2 / 20: 3$ alkenylacyl, $1 \cdot 10 \pm 0.33 \%$.

the mycelial cell walls without significant disruption of nuclei. As monitored by fluorescence microscopy and marker enzyme assays, the final preparations of purified nuclei were not contaminated by other organelles or membrane fragments including mitochondria, microsomes and plasma membranes. The marker enzyme assays used a minimum of 1000-fold more nuclei than were used for each electrospray MS analysis, and even using these much greater quantities of nuclei, there was no enzyme activity associated with other organelles in the nuclei preparations. Purity of a representative nuclear preparation was also confirmed by transmission electron microscopy.

Using GC-MS, it was confirmed that cholesterol, ergosterol and cholestane are incorporated by nuclei; presumably a proportion of these is located in the nuclear membrane. Although we did not quantify sterol uptake, when a roughly equal volume of nuclei was extracted from the three sterol-supplemented cultures, it was evident from the GC peak intensity that a significantly greater amount of cholestane was present in nuclei relative to nuclei from cells grown with cholesterol or ergosterol supplements. We did not rigorously monitor metabolism of the exogenous sterols, but there was no evidence of appreciable sterol metabolism from the GC chromatograms or GC-MS spectra.

The most unexpected finding in examining whole cell glycerophospholipid composition was the very high levels of ether-linked glycerophospholipids, especially in choline- and inositol-containing moieties. Comparable levels of alkyl-acyl and alkenyl-acyl moieties were found in nuclear membranes (Tables 1-4). There were statistically significant differences in many glycerophospholipid molecular species between whole cells and nuclei isolated from cells grown in the same medium.

The glycerophospholipid composition of nuclei isolated from cholestane-supplemented media for the three classes 


\section{Table 4. Glycerophosphoinositol molecular species from nuclei of $L$. giganteum grown on defined media}

Values are $(\mathrm{mol} \%)$ mean \pm SD from three separate cultures; figures in a given row with matching superscript letters are significantly different from one another $(P<0 \cdot 05)$. Values followed by an asterisk are significantly different in percentage composition from those found in total cell phospholipids ( $\mathrm{J}$. L. Kerwin and others, unpublished). Alk, alkyl-acyl molecular species; enyl, alkenyl-acyl species; all others are diacyl species. DM2, defined medium; DM2CHO, defined medium + cholesterol; DM2ERG, defined medium + ergosterol; $\mathrm{DM} 2 \mathrm{CHN}, \mathrm{DM} 2+$ cholestane; DM2SQN, DM2 + squalene.

\begin{tabular}{|c|c|c|c|c|c|}
\hline \multirow{2}{*}{$\begin{array}{l}\text { Molecular } \\
\text { species }\end{array}$} & \multicolumn{5}{|c|}{ Percentage composition after growth on: } \\
\hline & DM2 & DM2CHO & DM2ERG & DM2CHN† & DM2SQN \\
\hline $14: 0 / 18: 1$ alk & $4 \cdot 19 \pm 0 \cdot 37^{* a}$ & $2 \cdot 61 \pm 0 \cdot 15^{* a}$ & $3 \cdot 71 \pm 1 \cdot 04$ & $1 \cdot 61 \pm 0 \cdot 19^{* a, b}$ & $5 \cdot 00 \pm 1 \cdot 03^{* b}$ \\
\hline $18: 0 / 14: 0$ alk & $0.66 \pm 0.45$ & $0 \cdot 28 \pm 0 \cdot 06^{* a}$ & $0 \cdot 58 \pm 0 \cdot 16^{* b}$ & $0^{* a, b, c}$ & $0 \cdot 78 \pm 0 \cdot 30^{c}$ \\
\hline $16: 0 / 16: 0$ & $1 \cdot 58 \pm 0 \cdot 09^{a}$ & $1 \cdot 80 \pm 1 \cdot 07$ & $2 \cdot 88 \pm 1 \cdot 84$ & $0 * a, b$ & $2 \cdot 63 \pm 0 \cdot 55^{b}$ \\
\hline $18: 1 / 16: 1$ alk & $0 \cdot 22 \pm 0 \cdot 01^{a}$ & $1 \cdot 07 \pm 0 \cdot 27^{* a, b, c}$ & $0 \cdot 25 \pm 0 \cdot 15^{b}$ & $2 \cdot 37 \pm 0 \cdot 26^{* a, b, c}$ & $0.23 \pm 0.03 * c$ \\
\hline $18: 2 / 16: 0$ alk & $0 \cdot 11 \pm 0 \cdot 01^{a, c}$ & $0 \cdot 51 \pm 0 \cdot 13^{* a, b, d}$ & $0 \cdot 13 \pm 0 \cdot 07^{b}$ & $0 \cdot 18 \pm 0 \cdot 02^{c}$ & $0 \cdot 12 \pm 0 \cdot 02^{* d}$ \\
\hline $16: 0 / 18: 2$ alk & $0 \cdot 44 \pm 0 \cdot 03^{a, c}$ & $2 \cdot 14 \pm\left(1 \cdot 54^{* a, b, d}\right.$ & $0 \cdot 51 \pm 0 \cdot 30^{b}$ & $0 \cdot 62 \pm 0 \cdot 14^{c}$ & $0.46 \pm 0.06^{* a}$ \\
\hline $16: 1 / 18: 1$ alk & $0 \cdot 39 \pm 0 \cdot 02^{a}$ & $1.92 \pm\left(1 \cdot 48^{* a, b, c}\right.$ & $0.44 \pm 0 \cdot 26^{b}$ & $0^{* a, c}$ & $0 \cdot 41 \pm 0.06^{* c}$ \\
\hline $18: 0 / 16: 1$ alk & $0 \cdot 78 \pm 0 \cdot 07^{a}$ & $2 \cdot 22 \pm 1 \cdot 07$ & $0 \cdot 64 \pm 0.23 * b$ & $0^{* a, b, c}$ & $0 \cdot 83 \pm 0 \cdot 13^{* c}$ \\
\hline $18: 1 / 16: 0$ alk & $0.53 \pm 0.05^{a}$ & $1.25 \pm 0.60$ & $0 \cdot 44 \pm 0 \cdot 16^{* b}$ & $2 \cdot 39 \pm 0 \cdot 18^{* a, b, c}$ & $0.57 \pm 0.09 * c$ \\
\hline $18: 0 / 16: 0$ alk & $0 \cdot 47 \pm 0 \cdot 07^{a}$ & $1 \cdot 88 \pm 0.82$ & $0 \cdot 44 \pm 0 \cdot 18$ & $0 \cdot 13 \pm 0 \cdot 01^{* a}$ & $0 \cdot 87 \pm 0 \cdot 30$ \\
\hline $16: 0 / 18: 2$ & $0.54 \pm 0.06^{* a}$ & $2.57 \pm 0.35^{* a, b}$ & $0.83 \pm 0.04^{a}$ & $0^{* a, b}$ & $1 \cdot 04 \pm 0 \cdot 14^{* b}$ \\
\hline $16: 0 / 18: 1$ & $0 \cdot 26 \pm 0.05^{* a}$ & $1.50 \pm 0.79$ & $0.33 \pm 0.06^{b}$ & $0^{* a, b, c}$ & $0 \cdot 41 \pm 0 \cdot 11^{c}$ \\
\hline $16: 1 / 20: 4$ alk & $6 \cdot 80 \pm 0 \cdot 31^{* a}$ & $4 \cdot 22 \pm 1 \cdot 88$ & $5 \cdot 06 \pm 0 \cdot 63^{* b}$ & $3.37 \pm 0.77^{a, b}$ & $5 \cdot 37 \pm 0 \cdot 57$ \\
\hline $16: 0 / 20: 4$ alk & $6.86 \pm 0 \cdot 18^{* a, c}$ & $8 \cdot 60 \pm 2 \cdot 48$ & $7 \cdot 24 \pm 0 \cdot 24^{b, d}$ & $4 \cdot 36 \pm 0 \cdot 23^{a, b}$ & $5 \cdot 14 \pm 0 \cdot 26^{* c, d}$ \\
\hline $18: 0 / 18: 3$ alk & $1 \cdot 18 \pm 0 \cdot 19^{a}$ & $1.29 \pm 0.71$ & $1 \cdot 18 \pm 0.06^{b}$ & $0 * a, b, c$ & $1 \cdot 12 \pm 0 \cdot 23^{c}$ \\
\hline $18: 0 / 18: 2$ enyl & $2.63 \pm 0.43$ & $2 \cdot 87 \pm 1 \cdot 59$ & $2 \cdot 69 \pm 0 \cdot 21^{a}$ & $1 \cdot 26 \pm 0 \cdot 43^{a}$ & $2.50 \pm 0.51$ \\
\hline $18: 0 / 18: 2$ alk & $1 \cdot 76 \pm 0.08^{a}$ & $3 \cdot 85 \pm 1 \cdot 20^{c}$ & $1 \cdot 59 \pm 0 \cdot 34^{c}$ & $0^{* a, b, c}$ & $1 \cdot 16 \pm 0 \cdot 10^{a}$ \\
\hline $18: 0 / 18: 1$ enyl & $1.93 \pm 0.09^{a}$ & $4 \cdot 17 \pm 1 \cdot 30^{b}$ & $1 \cdot 74 \pm 0.38^{b}$ & $0^{* a, b}$ & $1 \cdot 27 \pm 0 \cdot 10^{a}$ \\
\hline $18: 0 / 18: 1$ alk & $1 \cdot 68 \pm 0 \cdot 18^{a}$ & $5 \cdot 16 \pm 2 \cdot 57$ & $2 \cdot 29 \pm 1 \cdot 15$ & $0 \cdot 29 \pm 0 \cdot 36^{a}$ & $1 \cdot 89 \pm 0 \cdot 42^{*}$ \\
\hline $16: 0 / 20: 4$ & $4 \cdot 32 \pm 0 \cdot 28^{a}$ & $2.97 \pm 1.76$ & $3 \cdot 91 \pm 0.25^{a}$ & $2 \cdot 79 \pm 0 \cdot 15^{a}$ & $5 \cdot 39 \pm 1 \cdot 17^{*}$ \\
\hline $18: 3 / 20: 4$ enyl & $3 \cdot 07 \pm 0 \cdot 65$ & $1 \cdot 77 \pm 0 \cdot 51^{a}$ & $2 \cdot 94 \pm 0.48^{*}$ & $2 \cdot 81 \pm 0 \cdot 30^{* b}$ & $4 \cdot 45 \pm 0 \cdot 55^{a, b}$ \\
\hline $18: 3 / 20: 4$ alk & $5.86 \pm 0.63$ & $3 \cdot 16 \pm 1 \cdot 60^{* a}$ & $4 \cdot 45 \pm 0.78^{b}$ & $7 \cdot 32 \pm 1 \cdot 95^{a}$ & $8 \cdot 13 \pm 1 \cdot 85^{b}$ \\
\hline $18: 2 / 20: 4$ alk & $7 \cdot 94 \pm 1 \cdot 24^{\alpha}$ & $5 \cdot 97 \pm 1 \cdot 49^{a}$ & $6 \cdot 22 \pm 1 \cdot 92$ & $5 \cdot 27 \pm 1 \cdot 24$ & $8 \cdot 11 \pm 0 \cdot 79 *$ \\
\hline $18: 1 / 20: 4$ alk & $7.91 \pm 0.65^{* a}$ & $6.67 \pm 2.06$ & $7 \cdot 35 \pm 1 \cdot 04$ & $4 \cdot 91 \pm 1 \cdot 03^{a}$ & $6.20 \pm 0.88$ \\
\hline $18: 0 / 20: 4$ alk & $5 \cdot 64 \pm 0 \cdot 28$ & $5 \cdot 17 \pm 1 \cdot 09^{a}$ & $6 \cdot 65 \pm 1 \cdot 42^{a}$ & $4.96 \pm 0.82$ & $4.92 \pm 0 \cdot 34$ \\
\hline $18: 2 / 20: 4$ & $2 \cdot 61 \pm 0 \cdot 28^{a}$ & $1.89 \pm 0.82 *$ & $2 \cdot 51 \pm 0.93$ & $1 \cdot 81 \pm 0 \cdot 30^{* a, b}$ & $3 \cdot 81 \pm 0 \cdot 26^{b}$ \\
\hline $18: 1 / 20: 4$ & $3 \cdot 11 \pm 0 \cdot 34^{a}$ & $1.66 \pm 0.60^{* a, b}$ & $4 \cdot 05 \pm 2 \cdot 28$ & $2.06 \pm 0.63^{c}$ & $3 \cdot 44 \pm 0 \cdot 13^{b, c}$ \\
\hline $18: 0 / 20: 4$ & $3.96 \pm 0.01^{a}$ & $1 \cdot 70 \pm 0 \cdot 27^{* a, b}$ & $3.69 \pm 0.77$ & $3.04 \pm 0.39^{b}$ & $3 \cdot 26 \pm 0 \cdot 21^{a}$ \\
\hline $20: 3 / 20: 4$ alk & $7 \cdot 27 \pm 0 \cdot 37 * a$ & $4.52 \pm 1.57$ & $5 \cdot 37 \pm 1 \cdot 54^{b}$ & $2 \cdot 97 \pm 0 \cdot 32^{a}$ & $7 \cdot 73 \pm 1 \cdot 41 * b$ \\
\hline $20: 0 / 20: 4$ enyl & $7 \cdot 85 \pm 0 \cdot 50^{a, b}$ & $7 \cdot 13 \pm 0 \cdot 85$ & $9 \cdot 09 \pm 1 \cdot 96^{c}$ & $5 \cdot 23 \pm 0 \cdot 61^{a}$ & $4 \cdot 27 \pm 0 \cdot 90^{b, c}$ \\
\hline $20: 0 / 20: 4$ alk & $5 \cdot 67 \pm 0.96$ & $6.05 \pm 1.66$ & $8 \cdot 47 \pm 2 \cdot 10$ & $4 \cdot 97 \pm 0.33^{a}$ & $5 \cdot 92 \pm 0.52^{a}$ \\
\hline $20: 0 / 20: 4$ & $1 \cdot 77 \pm 0.25$ & $1.45 \pm 0 \cdot 46^{* a}$ & $2.32 \pm 0.75$ & $2.43 \pm 0.57^{*}$ & $2.56 \pm 0.79 * a$ \\
\hline
\end{tabular}

† Other molecular species identified in nuclei from cholestane-supplemented media were: 14:0/16:0 alkyl-acyl, 1.04 $\pm 0 \cdot 14 \% ; 16: 1 / 16: 1$ alkenyl-acyl, $1 \cdot 57 \pm 0.25 \% ; 14: 0 / 18: 2$ alkenyl-acyl, $0 \cdot 22 \pm 0 \cdot 04 \% ; 14: 1 / 18: 2$ alkyl-acyl, $0 \cdot 22 \pm 0 \cdot 04 \% ; 18: 0 / 14: 0$ alkenyl-acyl, $0 \cdot 73 \pm 0 \cdot 08 \% ; 16: 0 / 16: 0$ alkenyl-acyl, $2 \cdot 93 \pm 0 \cdot 34 \% ; 14: 0 / 18: 0$ alkenyl-acyl, $1 \cdot 10 \pm 0 \cdot 13 \% ; 16: 0 / 16: 0$ alkyl-acyl, $1 \cdot 31 \pm 0 \cdot 63 \% ; 14: 0 / 18: 0$ alkyl-acyl, $1.39 \pm 0 \cdot 66 \% ; 16: 0 / 16: 1$ diacyl, $2 \cdot 12 \pm 0 \cdot 15 \% ; 16: 1 / 18: 0$ alkenyl-acyl, $1 \cdot 76 \pm 0 \cdot 19 \% ; 20: 0 / 14: 1$ alkyl-acyl, $0 \cdot 99 \pm 0 \cdot 07 \%$; 20:0/14:0 alkenyl-acyl, $0.77 \pm 0.06 \% ; 16: 0 / 18: 1$ alkyl-acyl, $0.56 \pm 0.04 \% ; 16: 1 / 18: 0$ alkyl-acyl, $1 \cdot 20 \pm 0.09 \% ; 16: 0 / 18: 0$ alkyl-acyl, $0 \cdot 24 \pm 0 \cdot 02 \% ; 16: 1 / 20: 4$ alkenyl-acyl, $2 \cdot 37 \pm 0 \cdot 32 \% 18: 1 / 18: 1$ alkenyl-acyl, $2 \cdot 39 \pm 0 \cdot 82 \% ; 18: 3 / 18: 0$ alkyl-acyl, $1 \cdot 40 \pm 0 \cdot 48 \% ; 18: 2 / 18: 0$ alkyl-acyl, $3 \cdot 66 \pm 0 \cdot 27 \% ; 18: 1 / 18: 0$ alkyl-acyl, $0 \cdot 29 \pm 0 \cdot 36 \% ; 20: 0 / 16: 0$ alkenyl-acyl, $1 \cdot 41 \pm 0 \cdot 25 \%$.

of lipid we examined was very different from that of nuclei isolated from mycelium grown in the other media (Tables 2-4). Nuclei from cholestane-supplemented cultures had seven GPC species not found, or present in very small amounts, in nuclei from cells grown in the other media (Table 2). Other significant differences found in cholestane-containing nuclei in this lipid class included elevated levels of 18:0/16:0 alkyl-acyl, 18:2/20:4 alkyl- acyl and 18:1/20:4 alkyl-acyl species; and a significant decrease in $18: 1 / 20: 4$ diacyl glycerophosphocholine. The most significant difference from nuclei isolated from the cholestane-supplemented cultures was the production of 22 GPI molecular species not present, or present in trace quantities, in nuclei from mycelia grown in the other media (Table 3). For the GPE species, cholestane supplementation resulted in significant increases in 
16:0/18:0 and 16:0/20:4 diacyl moieties, and large decreases in 18:1/20:4 and 18:2/20:4 diacyl molecular species (Table 4).

As was found for total cell fractions, ether-linked lipids made up a very high percentage of GPC and GPI molecular species in nuclear membranes (Table 1). Cholesterol supplementation of media produced the greatest change in ether-linked species between total cell and nuclear glycerophosphocholine species from L. giganteum grown on the same medium, with an average of $74 \%$ ether-linked species in the former and $58 \%$ in the latter.

\section{DISCUSSION}

One effect of isoprenoid supplementation of L. giganteum culture media is to alter the relative composition of mycelial and nuclear glycerophospholipid molecular species. This is not unexpected since sterols including cholesterol are readily incorporated into eukaryotic membranes, where they profoundly influence membrane integrity, fluidity and related physical properties (Bloch, 1983; Pasenkiewicz-Gierula et al., 1990, 1991; Bittman et al., 1992; Spector \& Yorek, 1985; Yeagle, 1989, 1990).

The most unexpected result of this investigation was the very high levels of GPC and GPI ether lipids. Oomycetes have long been considered a group of primitive fungi, but it is becoming clear that they evolved independently of true fungi. They are now considered to be related to heterokont algae in the Kingdom Chromista (CavalierSmith, 1989; Barr, 1992). In either case, there is limited information on the glycerophospholipid molecular species from these taxonomic groups that can be used for comparison. Ascomycete yeasts including Saccharomyces cerevisiae and Lipomyces starkeyi produce no or trace amounts of ether lipids (McElroy \& Stewart, 1967; Jollow et al., 1968). Higher quantities of ether-linked lipids (12\% GPC and 32\% GPE) were found in Pullularia pullulans (Dembitsky, 1989), but the few other yeasts that have been examined have insignificant quantities of this lipid class (Weete, 1980). Pythium prolatum, a sterol auxotroph related to L. giganteum, was found to produce $12-16 \%$ ether lipids in GPC and GPE fractions (Dembitsky, 1989).

Ether lipids are apparently synthesized by at least some members of all major kingdoms (Dembitsky, 1989). Archaeobacteria are characterized by production of etherlinked phytanyl isoprenoids rather than acylglycerols (de Rosa \& Gambacorta, 1988). Levels of ether lipids comparable to those found in L. giganteum have been documented in the anaerobic spirochaete Treponema byodysenteriae (Meyer \& Meyer, 1971); in the protozoan Paramecium tetraurelia, where these lipids make up $80 \%$ of the GPC fraction and $90-95 \%$ of ceramideaminoethylphosphonate (Kaneshiro, 1980); and in some major groups of the animal kingdom including Porifera (sponges), Mollusca and Echinodermata (Dembitsky, 1989). Ether-linked glycerophospholipids have been found in varying amounts in insects (Lambremont, 1972; Lambremont et al., 1976; Kulkarni et al., 1971), but we have found no reference to these compounds in mosquitoes, the host for L. giganteum.

L. giganteum and two genera of plant parasites in the Pythiaceae are the only documented sterol auxotrophs among Oomycetes. Elevated ether lipid content could be related to sterol auxotrophy. Ether-linked lipids generally have lower phase transition temperatures than the corresponding diacyl analogues (Lee \& Fitzgerald, 1980). Although there may be minimal differences in membrane properties induced following sterol binding to diacyl relative to ether-linked phospholipids (Bittman et al., 1984), it has been suggested that hydrogen bonding of cholesterol is enhanced in ether-linked glycerophospholipids relative to diacyl compounds due to the absence of a carbonyl at the $s n-1$ position of the former (Brockerhoff, 1974). The importance of sterols to the morphogenesis of L. giganteum and other sterol auxotrophs may have resulted in selection for a membrane glycerophospholipid composition with enhanced affinity for this class of compound.

Coordinated changes in isoprenoid supplementation or metabolism with fatty acid and phospholipid composition have been documented in fungi (Aaronson et al., 1982; Casey et al., 1992) and other organisms (Schmitz et al., 1990; Cinci et al., 1993; Maziere et al., 1990). It appears that isoprenoid and glycerophospholipid metabolism are also coordinated in L. giganteum. We have recently found that effects of cholesterol and other isoprenoids on $L$. giganteum morphogenesis are due in part to regulation of phospholipase $\mathrm{A}_{2}$ activity (MacKichan et al., 1994; unpublished observations), a pivotal class of enzyme involved in synthesis and remodelling of membranes. We are now investigating the interaction of sterols and glycerophospholipid metabolism in reproductively competent cultures.

\section{ACKNOWLEDGEMENTS}

This work was supported in part by a grant from the National Institutes of Health (5 RO1 AI22993) and by the Bosack and Kruger Charitable Foundation. We thank Lowell Ericsson, Biochemistry Department, University of Washington, for his assistance in operation of the electrospray mass spectrometer used in this investigation, and George Mueller, Botany Department, University of Washington, for performing the electron microscopy.

\section{REFERENCES}

Aaronson, L. R., Johnston, A. M. \& Martin, C. E. (1982). The effects of temperature acclimation on membrane sterols and phospholipids of Neurospora crassa. Biocbim Biopbys Acta 713, 456-462.

Barksdale, A. W. (1969). Sexual hormones of Achlya and other fungi. Science 166, 831-837.

Barr, D. J. S. (1992). Evolution and kingdoms of organisms from the perspective of a mycologist. Mycologia 84, 1-11.

Bittman, R., Clejan, S., Lund-Katz, S. \& Phillips, M. C. (1984). Influence of cholesterol on bilayers of ester- and ether-linked phospholipids - permeability and ${ }^{13} \mathrm{C}$-nuclear magnetic resonance measurements. Biochim Biophys Acta 772, 117-126. 
Bittman, R., Fugler, L., Clejan, S., Lister, M. D. \& Hancock, A. J. (1992). Interaction of cholesterol with conformationally restricted phospholipids in vesicles. Biocbim Biophys Acta 1106, 40-44.

Bloch, K. E. (1981). Sterol structure and membrane function. Curr Top Cell Reg 18, 289-299.

Bloch, K. E. (1983). Sterol structure and membrane function. Crit Rev Biochem 14, 47-92.

Brockerhoff, H. (1974). Model of interaction of polar lipids, cholesterol, and proteins in biological membranes. Lipids 9, 645-650.

Brunt, S. A. \& Silver, J. C. (1991). Molecular cloning and characterization of two distinct $b s p 85$ sequences from the steroid responsive fungus Achlya ambisexualis. Curr Genet 19, 383-388.

Casey, W. M., Keesler, G. A. \& Parks, L. W. (1992). Regulation of partitioned sterol biosynthesis in Saccharomyces cerevisiae. J Bacteriol 174, 7283-7288.

Cavalier-Smith, T. (1989). The kingdom Chromista. In The Chromophyte Algae: Problems and Perspectives, pp. 381-407. Edited by J. C. Green, B. S. C. Leadbeater \& W. L. Diver. Oxford: Clarendon Press.

Chapkin, R. S., Davidson, L. D. \& Davidson, L. A. (1992). Phospholipid molecular species composition of mouse liver nucleiinfluence of dietary $n-3$ fatty acid ethyl esters. Biochem $J$ 287, 237-240.

Chia, N.-C., Vilcheze, C., Bittman, R. \& Mendelsohn, R. (1993). Interactions of cholesterol and synthetic sterols with phosphatidylcholines as deduced from infrared $\mathrm{CH}_{2}$ wagging progression intensities. I Am Chem Soc 115, 12050-12055.

Cinci, G., Pagani, R., Pandolfi, M. L., Porcelli, B., Pizzichini, M. \& Marinello, E. (1993). Effects of testosterone on cholesterol levels and fatty acid composition in the rat. Life Sci 53, 91-97.

Davidson, S. M. K., Liu, Y. \& Regen, S. L. (1993). The influence of cholesterol on nearest-neighbor recognition in saturated phospholipid membranes. J Am Chem Soc 115, 10104-10110.

Dembitsky, V. M. (1989). Ether lipids of the organic world: formation and biotransformation. In Fats for the Future, pp. 173-188. Edited by R. C. Cambie. Chichester: Ellis Horwood.

Domnas, A. J. \& Warner, S. A. (1991). Biochemical activities of entomophagous fungi. Crit Rev Microbiol 18, 1-13.

Domnas, A. J., Srebro, J. P. \& Hicks, B. F. (1977). Sterol requirement for zoospore formation in the mosquito parasitizing fungus Lagenidium giganteum. Mycologia 69, 875-886.

Elliot, C. G. (1972). Sterols and the production of oospores by Phytophthora cactorum. J Gen Microbiol 72, 321-327.

Elliot, C. G. (1983). Physiology of sexual reproduction in Pbytophthora. In Phytophthora: Its Biology, Taxonomy, Ecology, and Pathology, pp. 71-80. Edited by D. C. Erwin, S. Bartnicki-Garcia \& P. H. Tsao. St. Paul, Minnesota: American Phytopathological Society.

Elliot, C. G. \& Sansome, E. (1977). The influence of sterols on meiosis in Phytophthora cactorum. J Gen Microbiol 98, 141-145.

Fiske, C. H. \& Subbarrow, Y. (1925). The colorimetric determination of phosphorus. J Biol Chem 66, 375-380.

Folch, J., Lees, M. \& Sloane-Stanley, G. H. (1957). A simple method for the isolation and purification of total lipids from animal tissues. $J$ Biol Chem 226, 497-509.

Gleason, F. H. (1968). Nutritional comparisons in the Leptomitales. Am J Bot 55, 1003-1010.

Horgen, P. A., Iwanochko, M. \& Bettiol, M. F. (1983). Antheridiol, RNA polymerase II and sexual development in the aquatic fungus, Achlya. Arch Microbiol 134, 314-319.
Jollow, D., Kellerman, G. M. \& Linname, A. W. (1968). The biogenesis of mitochondria. 3. The lipid composition of aerobically and anaerobically grown Saccharomyces cerevisiae as related to the membrane systems of cells. J Cell Biol 37, 221-230.

Kaneshiro, E. S. (1980). Positional distribution of fatty acids in the major glycerophospholipids of Paramecium tetraurelia. J Lipid Res 21, 559-570.

Kerwin, J. L. \& Duddles, N. D. (1989). Reassessment of the role of phospholipids in sexual reproduction by sterol-auxotrophic fungi. J Bacteriol 171, 3831-3839.

Kerwin, J. L. \& Washino, R. K. (1983). Sterol induction of sexual reproduction in Lagenidium giganteum. Exp Mycol 7, 109-115.

Kerwin, J. L., Duddles, N. D. \& Washino, R. K. (1991). Effects of exogenous phospholipids on lipid composition and sporulation by three strains of Lagenidium giganteum. J Invert Patbol 58, 408-414.

Kerwin, J. L., Tuininga, A. R. \& Ericsson, L. H. (1994). Identification of molecular species of glycerophospholipids and sphingomyelin using electrospray mass spectrometry. J Lipid Res 35, 1102-1114.

Ko, W. H. (1986). Stimulation of sexual reproduction of Pbytophthora cactorum by phospholipids. J Gen Microbiol 131, 2591-2594.

Kulkarni, A. P., Smith, E. \& Hodgson, E. (1971). The phospholipids of Manduca sexta tissues and the incorporation, in vivo, of ethanolamine, choline, and inorganic phosphate. Insect Biochem 1, 348-362.

Labremont, E. N. (1972). Ether-bonded lipids of insects : a quantitative comparison of the glyceryl ethers associated with ethanolamine and choline phosphoglycerides. Comp Biochem Physiol 41B, 337-342.

Labremont, E. N., Ferguson, J. R., III \& Greer, G. J. (1976). Confirmation of biosynthesis and partial structural characterization of the ether-linked ethanolamine phosphoglycerides of Heliothis. Insect Biochem 6, 363-366.

Lee, T. C. \& Fitzgerald, V. (1980). Phase transitions of alkyl ether analogs of phosphatidylcholine. Biochim Biophys Acta 598, 189-192.

Lorenz, R. T., Rodriguez, R. J., Lewis, T. A. \& Parks, L. W. (1986). Characteristics of sterol uptake in Saccharomyces cerevisiae. J Bacteriol 167, 981-985.

McElroy, F. A. \& Stewart, H. B. (1967). The lipids of Lipomyces lipofer. Can J Biochem 45, 171-178.

MacKichan, J. K., Tuininga, A. R. \& Kerwin, J. L. (1994). Preliminary characterization of phospholipase $A_{2}$ in Lagenidium giganteum. Exper Mycol 18, 180-192.

McMorris, T. C., Toft, D. O., Moon, S. \& Wang, W. (1993). Biological response of the female strain Acblya ambisexualis 734 to dehydro-oogoniol and analogues. Phytochemistry 32, 833-837.

Maziere, C., Auclair, M., Mora, L. \& Maziere, J.-C. (1990). Modification of phospholipid polar head group with monomethylethanolamine and dimethylethanolamine decreases cholesteryl ester and triacylglycerol synthesis in cultured human fibroblasts. Lipids 25, 311-315.

Meyer, H. \& Meyer, F. (1971). Lipid metabolism in the parasitic and free-living spirochetes Treponema pallidum (Reiter) and Treponema zuelzerae. Biochim Biophys Acta 231, 93-106.

Michell, R. H. \& Hawthorne, J. N. (1965). The site of diphosphoinositide synthesis in rat liver. Biochem Biophys Res Commun 21, 333-338.

Mrsny, R. J., Volwerk, J. J. \& Griffith, O. H. (1986). A simplified procedure for lipid phosphorus analysis shows that digestion rates vary with phospholipid structure. Chem Phys Lipids 39, 185-191.

Pasenkiewicz-Gierula, M., Subczynski, W. K. \& Kusumi, A. (1990). Rotational diffusion of a steroid molecule in phosphatidylcholine- 
cholesterol membranes: fluid-phase microimmiscibility in unsaturated phosphatidylcholine-cholesterol membranes. Biochemistry 29, 4059-4069.

Pasenkiewicz-Gierula, M., Subczynski, W. K. \& Kusumi, A. (1991). Influence of phospholipid unsaturation on the cholesterol distribution in membranes. Biochimie 73, 1311-1316.

Pinto, W. J., Lozano, R., Sekula, B. C. \& Nes, W. R. (1983). Stereochemically distinct roles for sterol in Saccharomyces cerevisiae. Biochem Biophys Res Commun 112, 47-54.

Rodriguez, R. J. \& Parks, L. W. (1983). Structural and physiological features of sterols necessary to satisfy bulk membrane and sparking requirements in yeast sterol auxotrophs. Arch Biochem Biophys 225, 861-871.

Rodriguez, R. J., Taylor, F. R. \& Parks, L. W. (1982). A requirement for ergosterol to permit growth of yeast sterol auxotrophs on cholestanol. Biochem Biophys Res Commun 106, 435-441.

Rodriguez, R. J., Low, C., Bottema, C. D. K. \& Parks, L. W. (1985). Multiple functions for sterols in Saccharomyces cerevisiae. Biochim Biophys Acta 837, 336-343.

de Rosa, M. \& Gambacorta, A. (1988). The lipids of archaebacteria. Prog Lipid Res 27, 153-175.

Schmitz, G., Beuck, M., Fischer, H., Mowicka, G. \& Robenek, H. (1990). Regulation of phospholipid biosynthesis during cholesterol influx and high density lipoprotein-mediated cholesterol efflux in macrophages. J Lipid Res 31, 1741-1752.

Smith, L. (1953). Spectrophotometric assay of cytochrome C oxidase. Methods Biochem Anal 2, 427-434.

Spector, A. A. \& Yorek, M. A. (1985). Membrane lipid composition and cellular function. $J$ Lipid Res 26, 1015-1035.

Van den Bossche, H. (1990). Importance and role of sterols in fungal membranes. In Biochemistry of Cell Walls and Membranes in Fungi, pp. 135-157. Edited by P. J. Kuhn, A. P. J. Trinci, M. J. Jung, M. W. Goosey \& L. G. Copping. Berlin: Springer-Verlag.

Warner, S. A. \& Domnas, A. J. (1981). Evidence for a cycloartenolbased sterol synthetic pathway in Lagenidium spp. Exp Mycol 5, 184-188.

Weete, J. D. (1980). Lipid Biochemistry of Fungi and Other Microorganisms, pp. 1-179. New York: Plenum Press.

Yeagle, P. L. (1989). Lipid regulation of cell membrane structure and function. FASEB J 3, 1833-1842.

Yeagle, P. L. (1990). Role of cholesterol in cellular functions. In Advances in Cholesterol Research, pp. 111-132. Edited by E. Esfahani \& J. B. Swaney. New Jersey: The Telford Press.

Received 13 June 1994; revised 29 September 1994; accepted 6 October 1994. 